\section{Vocational Identity Development and the Role of Human Resources Management. A Systematic Literature Review}

\section{Elia Pizzolitto}

M.Sc. Department of Business Economics University G. D'Annunzio-Chieti-Pescara, Italy

\section{Ida Verna}

$\mathrm{PhD}$. Department of Business Economics University G. D’Annunzio-Chieti-Pescara, Italy

Submitted: 20 October 2020

Accepted: 02 December 2020

Published: 31 December 2020

Corresponding author:

Elia Pizzolitto

DOI: 10.19044/esj.2020.v16n34p80

(c) Copyright 2020 Pizzolitto E. \& Verna I. Distributed under Creative Commons BY-NC-ND 4.0 OPEN ACCESS

\section{Abstract}

Objectives. The development and strengthening of employees' vocational identity are critical issues in organizations. The absence of professional selfawareness could negatively affect the emotional status of individuals. Moreover, such a condition can lead to a worsening of organizational performance. For this reason, studying the connections between human resources management and identity development is essential. Data and method. This exploratory study performs a systematic literature review starting from SCOPUS and EBSCO Host databases to understand how literature debates these connections. Results. The majority of extracted articles belong to the following fields: human resources management, psychology, and organizational studies. The connections between these three fields represent a clear theoretical framework for understanding how literature debates professional identity development in organizations. The theories considered in the extracted articles are numerous, but the social identity theory and the social categorization theory are the most cited by the authors. Four main themes emerge from the content analysis: motivation, satisfaction, and well-being of employees; the "crisis" status of human resource management; professional identity development in the workplace; the career adaptation of individuals. Conclusions. This study allows confirming how the development of professional identity is critical in organizations. Although the literature shows a considerable interest in vocational identity and human resources management, this study reflects on the possibility of producing further in-depth examinations concerning the connections

Pizzolitto E. \& Verna I., (2020). Vocational Identity Development and the Role of Human Resources Management. A Systematic Literature Review. European Scientific Journal, ESJ, 16(34), 80. 
between these topics. Further studies should explore vocational identity development through a renovated strategic role of human resources management, no more limited to a bureaucratic partner.

Keywords: HR; human resources; crisis; vocational identity; career adaptation

\section{Introduction}

Identity is a structure that defines the self-consciousness of an individual in the world (Erikson, 1963; 1968; 1982). Such structure depends on many domains, e.g., religious, social, or family (Grotevant, 1987). The vocational domain is the most important in the identity development process (Marcia, 1966; 1967; 1980; Porfeli et al., 2011). The development of individuals' vocational identity is firmly connected to their professional contexts. Moreover, it is related to every other identity domain. Satisfaction, motivation, and well-being are influenced by the degree of self-awareness achieved by a person (Guan et al., 2015). Although self-consciousness has many different origins, the most critical one is individuals' perception of how they identify with their professions (Horton et al., 2014).

Building individuals' identity in their organizations, workgroups, and careers is a fundamental element of their professional development. The lack of self-awareness could bring complex pathologies, e.g., anxiety, depression, and burnout, which could generate extremely negative impacts on organizational performances (Steffens et al., 2017). For this reason, understanding the individuals' reactions to their duties and working environments is fundamental. People's perception about their vocational identity could depend on the quality and the variety of their duties (Katz, 1978; Mooney et al., 2016), their hierarchical position (Horton et al., 2014), and their moral and religious values (Hèliot et al., 2020). The achievement of selfawareness has a strong impact on the organizational identification of individuals.

In particular, the literature underlines that reaching vocational identity is critical for creating value in organizations (Armishaw, 2007; Brown, 2015; Klotz et al., 2014). In this sense, human resources (HR) management has a fundamental role in promoting (or limiting) the achievement of vocational self-awareness (Roberts et al., 2005).

An obstacle to this direction can be represented by the particular historical moment that Human Resources (HR) management is experimenting. The professional identity of HR is suffering from the complexities of the labor market. These conditions, exacerbated by the Covid-19 pandemic and the contemporary concepts of professions and labor, convert HR management's nature, highlighting a moment of "crisis" in this function (Mackay, 2017; Wright, 2008). The conservative idea that associates HR management with an administrative and technical corporate function only linked to personnel supervision and development is outdated and risks transforming HR 
management into a semi-profession (Mackay, 2017; Wright, 2008). Therefore, the HR function's identity is trying to evolve from an administrative and bureaucratic department to a strategic partner for the firm (Hallier \& Summers, 2011; Pritchard \& Fear, 2015; Roche \& Teague, 2012; Wright, 2008). In this regard, the literature observed that the HR function has to consider both the employees and the top management's needs. Therefore, it experiments a contradictory position (Mackay, 2017). “The HR function's status and impact are often subjected to the whims of senior and line management and the vagaries of the economic climate" (Hallier \& Summers, 2011).

The identity "crisis" experimented by HR function could find a solution in employees' professional identity formation. As previously observed, the HR function could assume a renewed strategic role in organizations, focusing on developing employees' professional identity. In this sense, this article analyzes the research on identity topic, both from a professional viewpoint and for the HR function. Simultaneously, the paper proposes a reflection on the possible connections between professional identity development and the HR function role.

In this regard, following Denyer and Tranfield (2009), this study performs a systematic literature review (SLR) to create a general intersection framework between the professional identity and the HR management role.

In particular, the article is organized as follows. The first section performs a detailed explanation of methodology, research questions, algorithm for articles extraction, and analysis process. In the second section, this study performs a descriptive analysis of the extracted articles. In the third section, the articles' contents are analyzed, with specific attention to the papers' theories and the emerging themes. The paper finds its conclusions with critical reflections on the research questions and opportunities for further research development in this field.

\section{Methodology}

This article aims to achieve a better knowledge of the connections between identity and HR management. This paper is not referring only to employees' identity but also HR departments' professional identity. Identity development is one of the most important topics in the philosophical and psychological sciences. Therefore, the research involved both conceptual and empirical articles.

\section{1 - Choice of methodology: the systematic literature review}

The methodology chosen to perform this study is the systematic literature review (SLR). This methodology aims to identify the higher-quality papers related to a specific topic, laying the foundation for further research (Petticrew \& Roberts, 2006), through a detailed description of the employed 
procedure from data extraction to contents analysis (de Zubielqui, Fryges, \& Jones, 2019). This process has several advantages concerning reliability issues and compared to other literature review processes, e.g., narrative (Fink, 2013). This study performs the SLR through the procedure designed by Grant and Booth (2009), with the initial formulation of research questions (Figure 1).

Figure 1 - Phases of SLR employed in this study

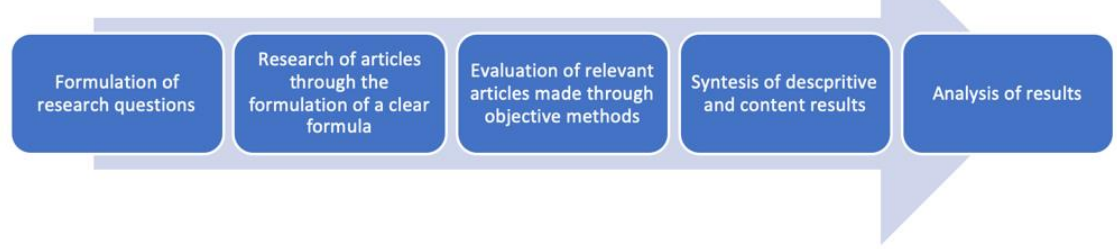

Source: Grant and Booth (2009) with the addition of research questions formulation

\section{2 - Research questions}

Identity development is firmly connected to organizations, especially in the light of critical changes in advanced Countries' economies experimented during the last 50 years. In fact, moving from physical production to service provision, the attention to the professional development of employees, talent research, and focus on performance are fundamental topics in any scientific and practical discussion.

To reach a complete comprehension of the existing debate on identity ascribed to organizations, to identify the existence or absence of a solid fundamental theory on the subject, to identify gaps in the literature, and to propose new points of scientific reflection, this article proposes the following three research questions:

RQ1: How literature debates the vocational identity topic in organizations? RQ2: How literature debates the identity of the HR function?

RQ3: What intersections exist between the development of employees' professional identity and the HR function's identity?

\section{3 - Data extraction and results filtering}

Databases employed for the articles extraction were SCOPUS and EBSCO Host. The first extraction was performed by considering the title, the abstract, and the articles' keywords. The formulas employed in SCOPUS and EBSCOHost is shown in Table 1. The terms "vocational identity", "professional identity", and "occupational identity" are used as synonyms in literature and are often associated with "career identity" (Henry, 1993; Smitina, 2010). 
Table 1 - Formula used for articles research in SCOPUS and EBSCOHost

\begin{tabular}{|c|c|}
\hline SCOPUS & EBSCOHost \\
\hline $\begin{array}{c}\text { TITLE-ABS-KEY (("human resourc*" OR } \\
\text { "personnel") AND ("vocation*" OR } \\
\text { "occupation*" OR "profession*" OR } \\
\text { "career") AND "identity"). }\end{array}$ & $\begin{array}{c}\text { (("human resourc*" OR "personnel") AND } \\
\text { ("vocation*" OR "occupation*” OR } \\
\text { "profession*" OR "career") AND } \\
\text { "identity") }\end{array}$ \\
\hline
\end{tabular}

The research was performed in September 2020, without time restrictions. The graphic representation of this process, from the initial extraction to the final group of selected articles, is shown in Figure 2.

Figure 2 - Extraction process and results limitation

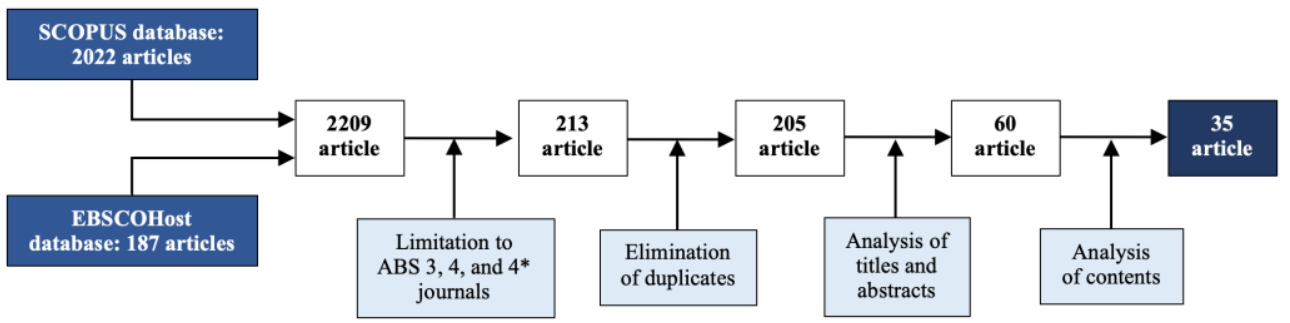

\section{Descriptive analysis of extracted articles}

\section{1 - Publication year and papers typology}

Following Vrontis and Christofi (2019) and Danese et al. (2018), a graph was drawn - Figure 3 - for the time distribution of the publications analyzed in this study, with the distinction between theoretical and empirical papers. The 35 articles were divided into 29 empirical papers (82.86\%) and 6 theoretical papers $(17.14 \%)$. The interest in this topic increased over time. In particular, recent literature seems more concentrated on empirical contributions. One possible reason is that overall identity, professional identity, and social identity have been broadly debated in the literature. Nevertheless, this study calls for additional theoretical contributions.

\section{2 - Journals, field, and citations}

The journals extracted for this study showed an interesting combination of scientific fields (Table 1; Figure 4). The larger number of articles was identified in the HR management and employment studies field (14 articles; 40\%). The following fields are organizational psychology (7 articles; 20\%), organizational studies (5 articles; 14.29\%), general management, ethics, and corporate social responsibility (3 articles; $8.57 \%$ ). Combining these fields showed an effective framework for the debate about 
vocational identity in organizations and conferred a considerable level of validity to this analysis.

The most cited articles belonged to the organizational psychology field, with 421 citations (Alvesson, 2001), 392 citations (Blader \& Tyler, 2009) and 334 citations (Chan \& Drasgow, 2001), general management, ethics, and corporate social responsibility field, with 180 citations (Morgan et al., 2005) and human resource management and employment studies field, with 178 citations (Dries, 2013).

\section{3 - Number and origins of authors per article}

This study referred to the SCOPUS database to analyze authors' provenience per extracted article, depending on the year of publication. UK had the greater number of authors $(23 ; 27.71 \%)$, followed by the USA (18 authors; $21.69 \%$ ), Australia and Finland (both with 6 authors, $7.23 \%$ ), Taiwan (5 authors; $6.02 \%$ ), and Canada (4 authors, $4.82 \%$ ). China, Germany, and New Zealand contributed with 3 authors for each Country (3.61\%). Hong Kong, Netherlands, Ireland, and Spain contributed with 2 authors for each Country $(2.41 \%)$. Finally, Belgium, Ireland (UK), Singapore, and Sweden contributed with 1 author for each Country (1.20\%). To be specific, 5 of the 6 authors from Finland were publishing the same article (Björkman et al., 2013).

The number of authors per extracted article was also checked through the SCOPUS database. Before the year 2000, the average number of authors for the extracted articles was 1.50. For the first decade after 2000, this average increase to 1.92. From 2011 to 2020, the average number of authors of extracted articles was 2.86. Therefore, the number of authors has been increasing during the period considered.

Table 1 - Journals, fields and ranking of extracted articles

\begin{tabular}{|l|c|l|c|c|}
\hline \multicolumn{1}{|c|}{ Journal } & $\begin{array}{c}\text { Number } \\
\text { of } \\
\text { articles }\end{array}$ & \multicolumn{1}{|c|}{ Field (ABS)* } & $\begin{array}{c}\text { Ran } \\
\text { king }\end{array}$ & Citations \\
\hline Journal of Applied Psychology & 2 & PSYCH (WOP-OB) & $4^{*}$ & 726 \\
\hline Human Relations & 5 & ORG STUD & 4 & 548 \\
\hline $\begin{array}{l}\text { Human Resource Management } \\
\text { Review }\end{array}$ & 2 & HRM\&EMP & 3 & 262 \\
\hline Academy of management review & 1 & ETHICS-CSR-MAN & $4^{*}$ & 180 \\
\hline Journal of Management Studies & 1 & ETHICS-CSR-MAN & 4 & 174 \\
\hline Administrative science quarterly & 1 & ETHICS-CSR-MAN & $4^{*}$ & 158 \\
\hline Human Resource Management & 4 & HRM\&EMP & 4 & 133 \\
\hline Journal of Vocational Behavior & 2 & PSYCH (WOP-OB) & 4 & 120 \\
\hline $\begin{array}{l}\text { Human Resource Management } \\
\text { Journal }\end{array}$ & 4 & HRM\&EMP & 4 & 66 \\
\hline Journal of Organizational Behavior & 1 & PSYCH (WOP-OB) & 4 & 60 \\
\hline Computers in Human Behavior & 1 & INFO MAN & 3 & 55 \\
\hline Journal of marketing & 1 & MKT & $4 *$ & 38 \\
\hline
\end{tabular}




\begin{tabular}{|c|c|c|c|c|}
\hline $\begin{array}{llr}\begin{array}{l}\text { Economic } \\
\text { Democracy }\end{array} & \text { and } & \\
\end{array}$ & 1 & HRM\&EMP & 3 & 26 \\
\hline $\begin{array}{lr}\text { International } & \text { journal of } \\
\text { Contemporary } & \text { Hospitality } \\
\text { Management } & \\
\end{array}$ & 1 & SECTOR & 3 & 21 \\
\hline Work, Employment and Society & 1 & HRM\&EMP & 4 & 15 \\
\hline $\begin{array}{l}\text { International Journal of Human } \\
\text { Resource Management }\end{array}$ & 1 & HRM\&EMP & 3 & 14 \\
\hline $\begin{array}{l}\text { Journal of Occupational Health } \\
\text { Psychology }\end{array}$ & 1 & PSYCH (WOP-OB) & 4 & 11 \\
\hline $\begin{array}{l}\text { New Technology, Work and } \\
\text { Employment }\end{array}$ & 1 & HRM\&EMP & 3 & 9 \\
\hline $\begin{array}{lll}\text { Technological Forecasting and } \\
\text { Social Change }\end{array}$ & 1 & SOC SCI & 3 & 6 \\
\hline Studies in Higher Education & 1 & MDEV\&EDU & 3 & 4 \\
\hline Journal of Managerial Psychology & 1 & PSYCH (WOP-OB) & 3 & 1 \\
\hline Strategic Management Journal & 1 & STRAT & $4^{*}$ & 0 \\
\hline \multicolumn{5}{|l|}{ * } \\
\hline $\begin{array}{l}\text { HRM\&EMP: Human Resource Man } \\
\text { PSYCH (WOP-OB): Psychology (O1 } \\
\text { ETHICS-CSR-MAN: General Mana } \\
\text { INFO MAN: Information System an } \\
\text { MDEV\&EDU: Management Develo } \\
\text { MKT: Marketing } \\
\text { SECTOR: Sectoral Studies } \\
\text { SOC SCI: Social Sciences } \\
\text { ORG STUD: Organization Studies } \\
\text { STRAT: Strategic Management }\end{array}$ & $\begin{array}{l}\text { men } \\
\text { iizat } \\
\text { lent, } \\
\text { ana } \\
\text { ent a }\end{array}$ & $\begin{array}{l}\text { d Employment Studies } \\
\text { l) } \\
\text { iics and Corporate Soc } \\
\text { ent } \\
\text { Education }\end{array}$ & $1 \mathrm{Re}$ & bility \\
\hline
\end{tabular}

Figure 3 - Publication years and papers typology

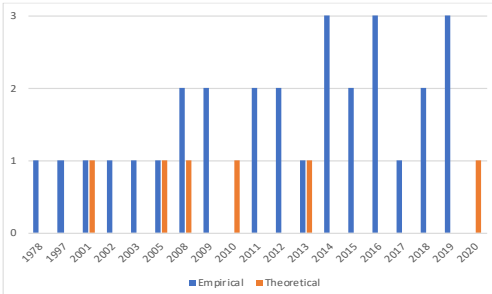

Figure 5 - Provenience of authors of extracted articles

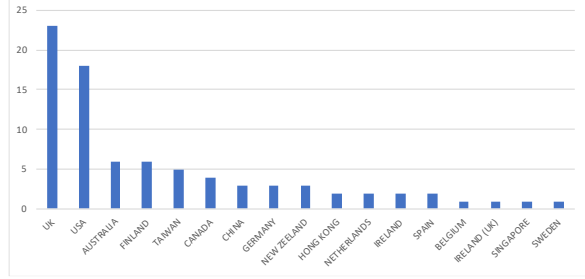

Figure 4 - Percentage distribution of extracted articles based on scientific field

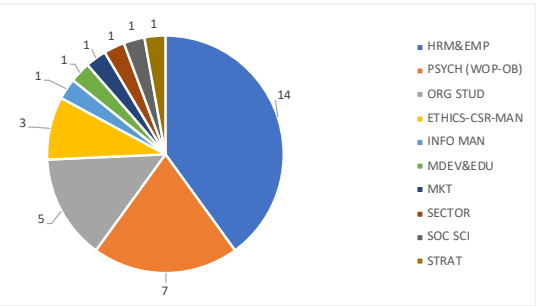

Figure 6 - Number of authors per extracted article

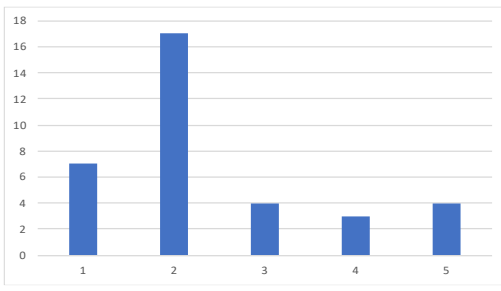




\section{4 - Content analysis}

\section{1 - Theories, methods, and statistical units}

In this paragraph, the main theories cited in the extracted articles, the methods used for data gathering, their main findings, and the typologies of statistical units are considered.

The most cited theories in the extracted articles were the social identity theory (12 articles, $34.28 \%)$, the social categorization and the selfcategorization theory (7 articles, 20\%), the cognitive dissonance theory, and the social exchange theory were cited in 2 articles $(5.51 \%)$. The other 24 cited theories were identified (Table 2). 
Table 3 - Articles types, methods, samples and Countries considered in the samples

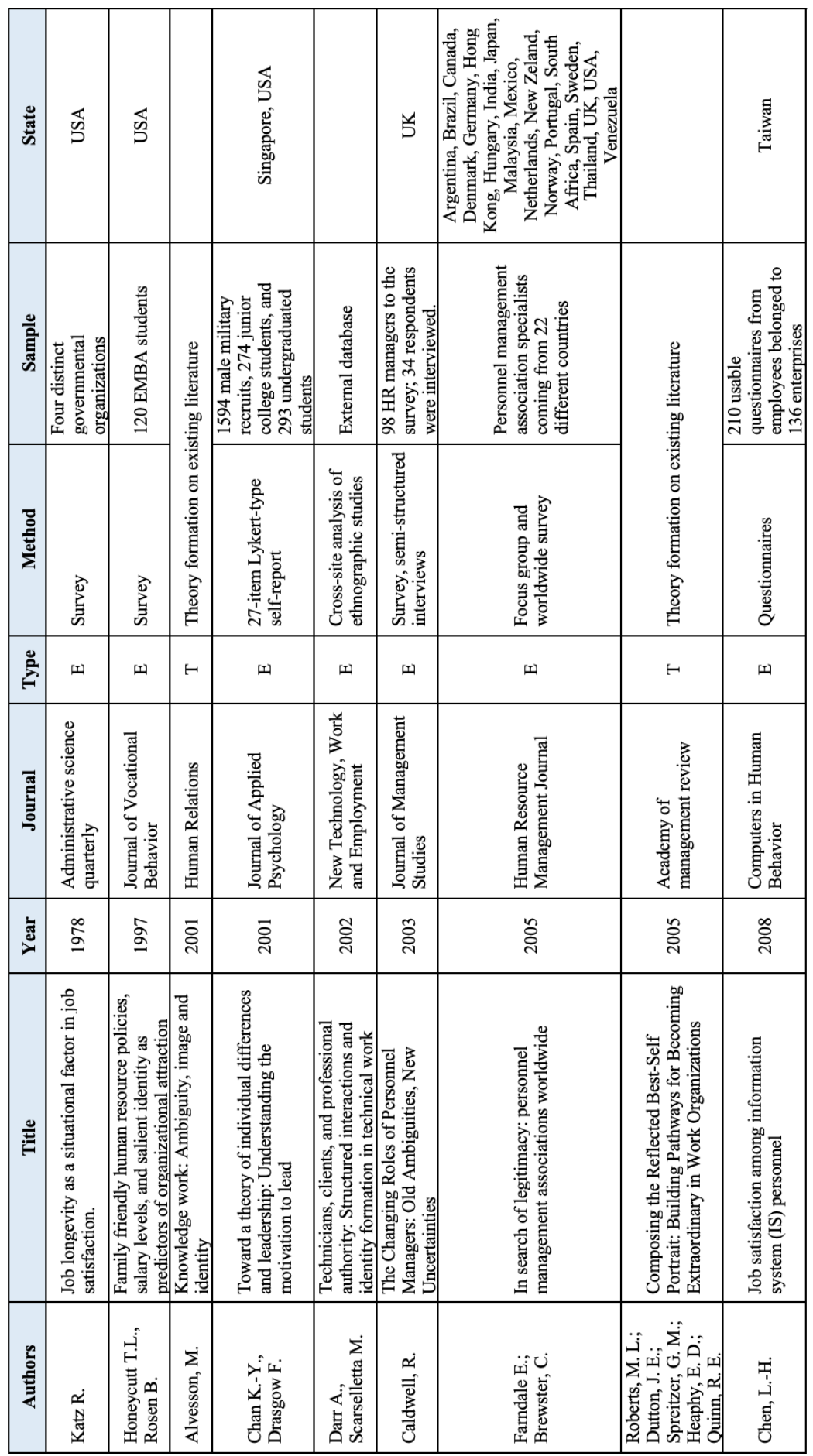




\begin{tabular}{|c|c|c|c|c|c|c|c|c|c|}
\hline 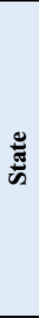 & 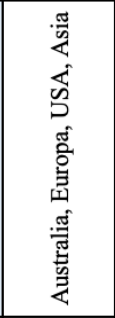 & & 苑 & 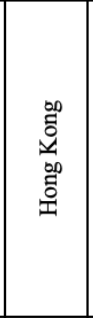 & & 歺 & b & $\begin{array}{l}\text { ్ㅠ } \\
\text { 总 }\end{array}$ & 崩 \\
\hline & 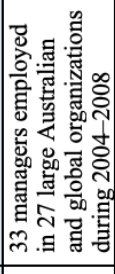 & & 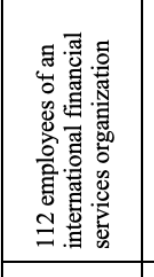 & 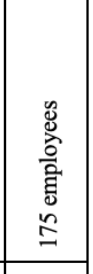 & 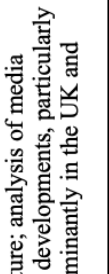 & 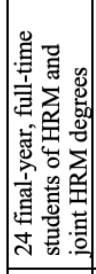 & 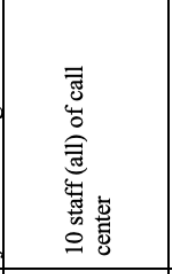 & 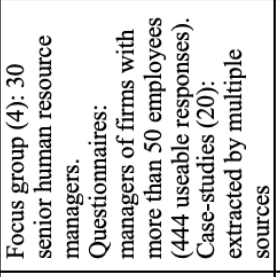 & 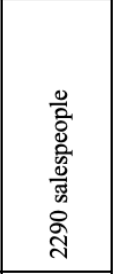 \\
\hline 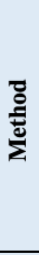 & 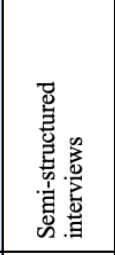 & 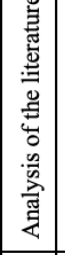 & 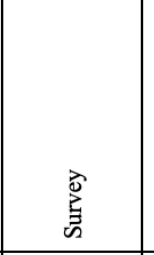 & 密 & 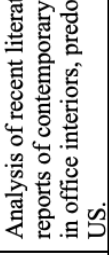 & 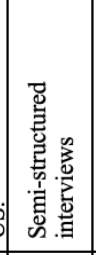 & 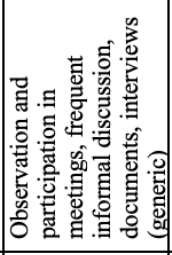 & 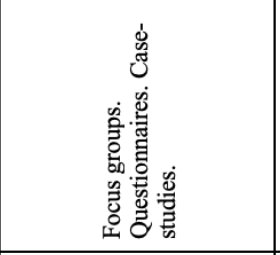 & 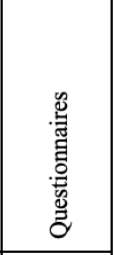 \\
\hline 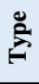 & 피 & $H$ & 피 & 피 & $H$ & 피 & 피 & 피 & 피 \\
\hline 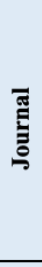 & 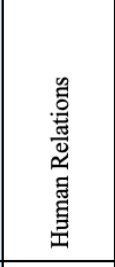 & 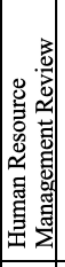 & 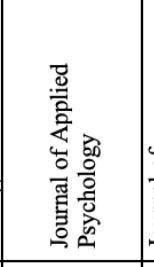 & 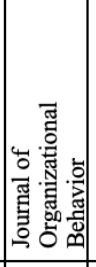 & 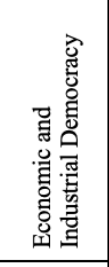 & 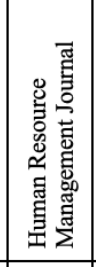 & 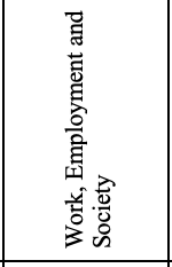 & 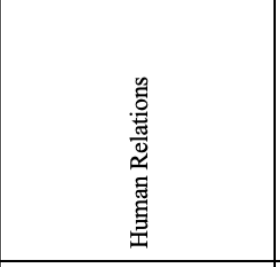 & 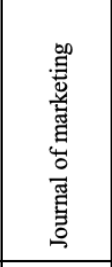 \\
\hline 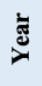 & 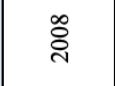 & 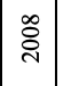 & ઠ્તે & ठ્సે & $\stackrel{\text { ते }}{\text { ते }}$ & $\overline{\vec{\sim}}$ & $\overline{\vec{乛}}$ & సี & $\stackrel{\sim}{\sim}$ \\
\hline$\stackrel{\Xi}{\Xi}$ & 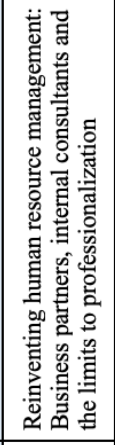 & 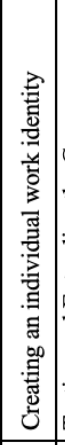 & 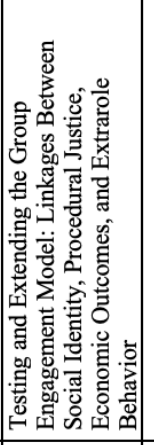 & 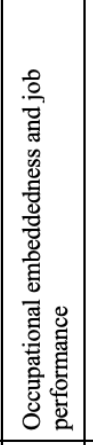 & 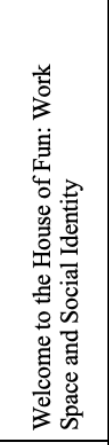 & 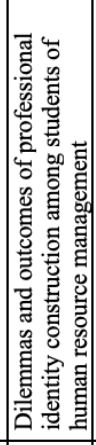 & 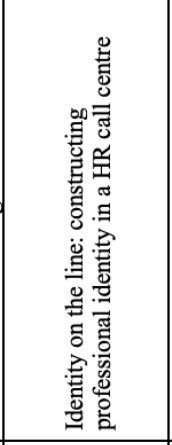 & 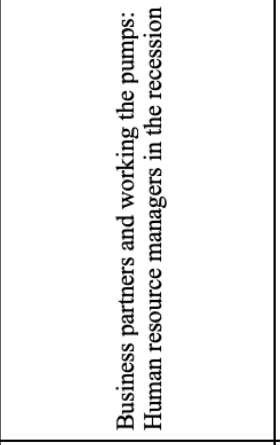 & 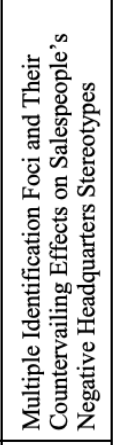 \\
\hline $\overrightarrow{\mathrm{E}}$ & 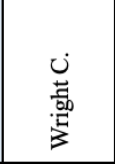 & 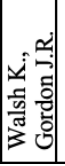 & 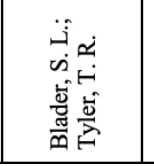 & 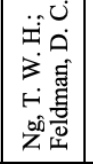 & 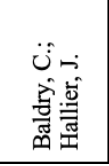 & 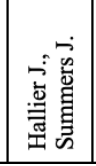 & 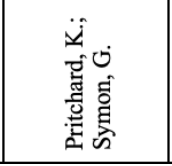 & 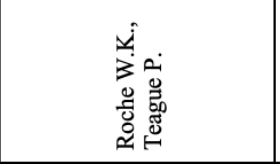 & 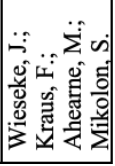 \\
\hline
\end{tabular}




\begin{tabular}{|c|c|c|c|c|c|c|c|c|c|c|}
\hline 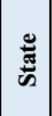 & 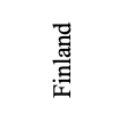 & & b & 票 & b & $\breve{s}$ & 襡 & 䍖 & 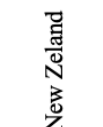 & $\begin{array}{l}\text { 量 } \\
\text { की }\end{array}$ \\
\hline 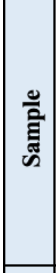 & 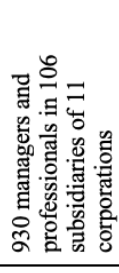 & 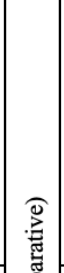 & 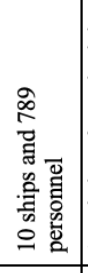 & 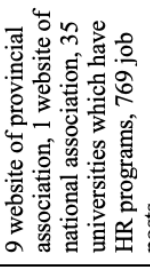 & 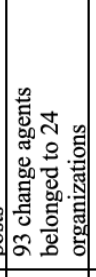 & 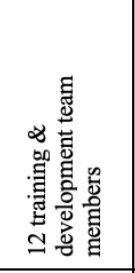 & 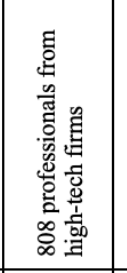 & 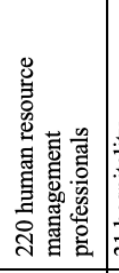 & 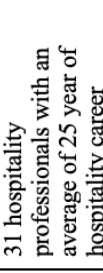 & 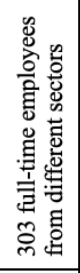 \\
\hline 总 & 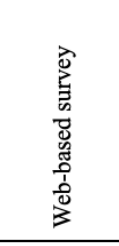 & 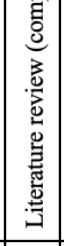 & 密 & 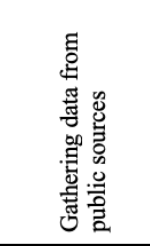 & 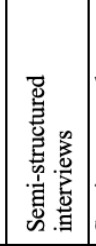 & 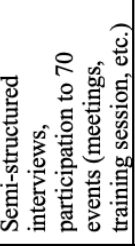 & 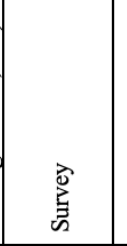 & $\begin{array}{l}\text { 离 } \\
\text { 空 }\end{array}$ & 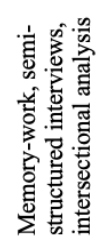 & 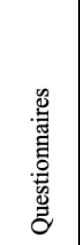 \\
\hline$\stackrel{0}{2}$ & w & $H$ & 山 & (1) & 山 & w & س & w & 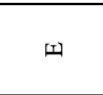 & \pm \\
\hline $\begin{array}{c}\overline{\mathrm{g}} \\
\overline{\mathrm{g}} \\
\overline{\mathrm{z}}\end{array}$ & 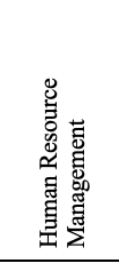 & 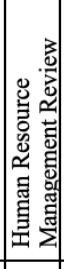 & 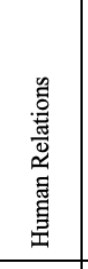 & 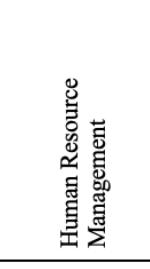 & 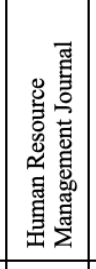 & 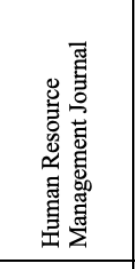 & 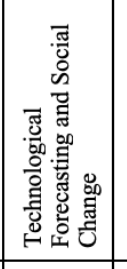 & 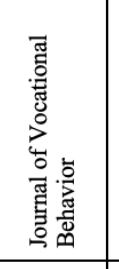 & 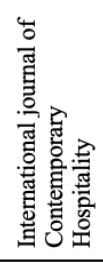 & 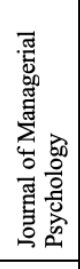 \\
\hline$\underset{\searrow}{\stackrel{\Xi}{\sharp}}$ & $\stackrel{m}{\stackrel{\sim}{*}}$ & $\stackrel{m}{\grave{c}}$ & $\stackrel{\Xi}{\stackrel{\sim}{~}}$ & ث̈ & 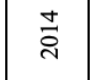 & $\stackrel{\text { กิ }}{n}$ & $\stackrel{n}{\tilde{N}}$ & :ั & 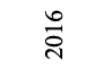 & $\stackrel{\text { : }}{\circ}$ \\
\hline 苂 & 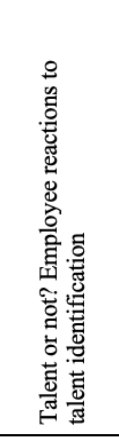 & 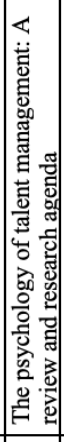 & 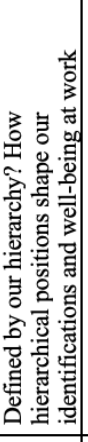 & 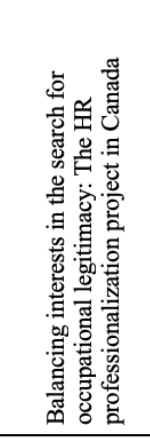 & 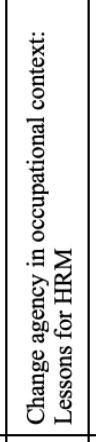 & 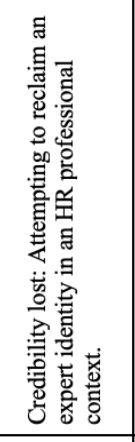 & 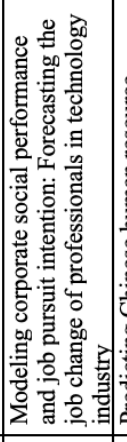 & 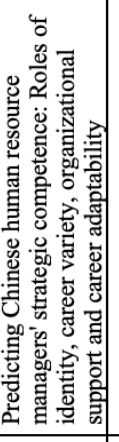 & 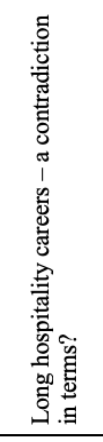 & 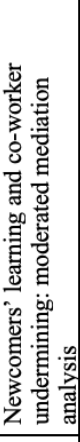 \\
\hline 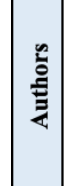 & 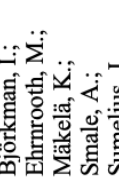 & 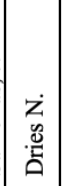 & 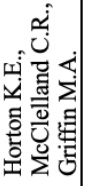 & 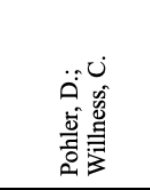 & 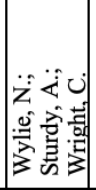 & 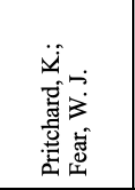 & 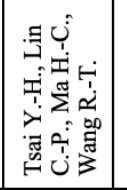 & 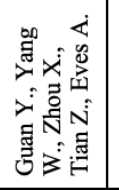 & 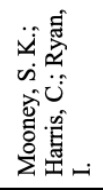 & 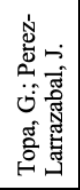 \\
\hline
\end{tabular}


December 2020 edition Vol.16, No.34

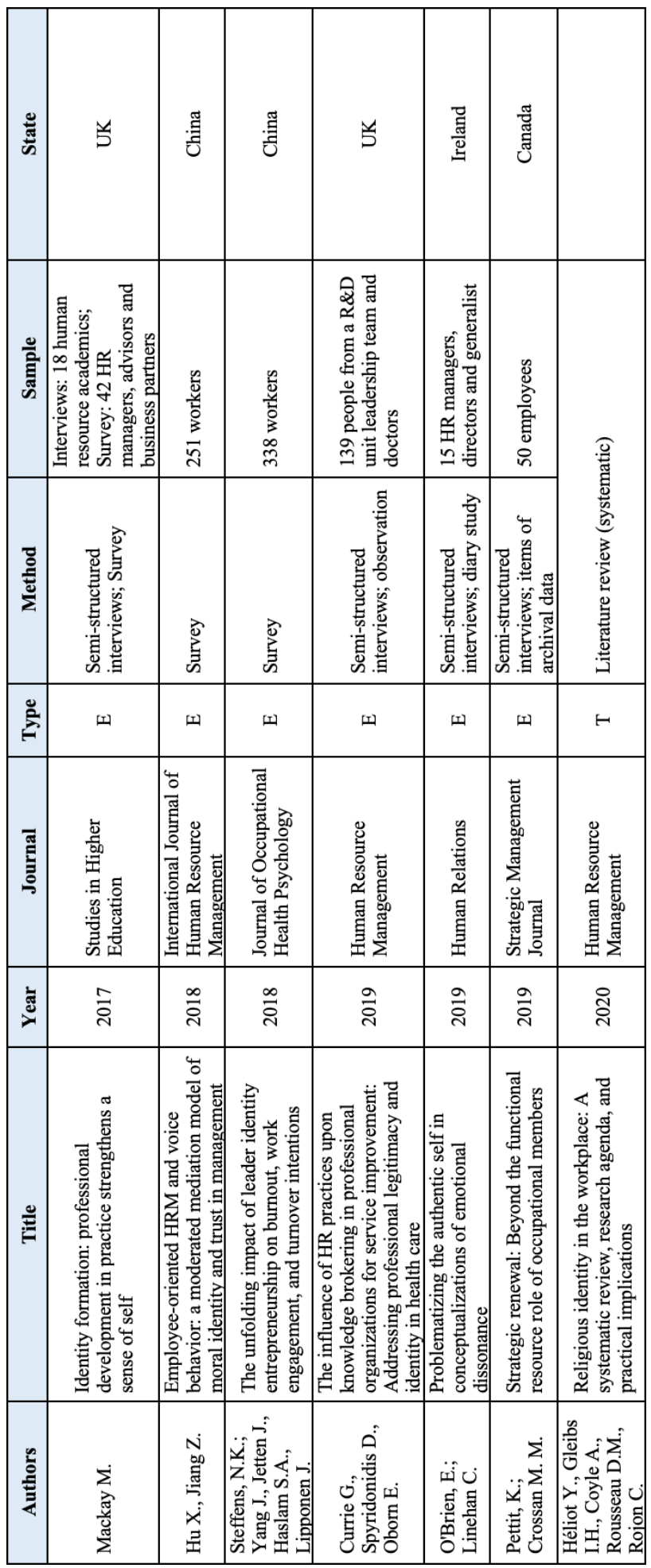


In Table 3, methods, numerosity of samples, and Countries considered for sampling in the selected articles are shown. In particular, 19 different gathering data methods were detected during the content analysis of the extracted articles. The most popular method was the survey, used by 13 articles $(37.14 \%)$, followed by semi-structured interviews (10 articles, $28.57 \%$ ), literature analysis (5 articles, $14.28 \%$ ), questionnaires ( 3 articles, $8.57 \%$ ), and focus groups ( 2 articles, $5.71 \%$ ). All other methods were used in 1 article. Several papers used combined methods for gathering data. Often, semi-structured interviews were combined with other methods (Mooney et al., 2016; O’Brien \& Linehan, 2019; Pettit \& Crossan 2019) (Figure 7).

During the content analysis, 31 typologies of statistical units were identified (29 articles). Employees were the most popular statistical units, used by 9 articles (31.03\%), followed by managers and directors, used by 7 articles (24.14\%) and specialists, used by 5 articles (17.24\%). Students, organizational institutions, and professional associations were used by 3 articles (10.34\%). Workers were used by 2 articles (6.7\%). Finally, academics and external sources of information were used by 1 article. In Figure 8, a graph was drawn to summarize the typology of statistical units identified in the extracted articles.

Most of the statistical units used in the 19 empirical papers came from the UK ( 8 articles, $42.11 \%$ ), from the USA (7 articles, 36.84\%). China followed with 3 papers (15.79\%). Statistical units from Ireland, Taiwan, and Canada were used by 2 articles $(10.53 \%)$. Other provenience of statistical units used in the extracted articles were Australia, Singapore, Finland, Hong Kong, Spain, and New Zealand. In particular, Farndale and Brewster (2005) performed remarkable research used a worldwide sample for its survey. In Figure 9, the provenience of statistical units is shown.

Finally, in Table 4, the summary of the extracted articles' main findings is shown.

Table 2 - Cited theories in the extracted articles

\begin{tabular}{|c|c|c|}
\hline Theories & Extracted articles & $\begin{array}{l}\text { References cited in extracted } \\
\text { papers }\end{array}$ \\
\hline Broaden-and-build theory & Roberts et al. (2005) & Fredrickson (2000) \\
\hline Career construction theory & Guan et al. (2016) & Savickas $(2005 ; 2013)$ \\
\hline Career Theory & $\begin{array}{l}\text { Mooney, Harris \& Ryan } \\
\text { (2016) }\end{array}$ & Sullivan and Baruch's (2009) \\
\hline \multirow[b]{2}{*}{ Cognitive dissonance theory } & Björkman et al. (2013) & Festinger (1957) \\
\hline & $\begin{array}{|lll|}\begin{array}{l}\text { O'Brien } \\
(2019)\end{array} & \& & \text { Linehan } \\
\end{array}$ & Aronson et al. (1999) \\
\hline Construal-level theory & Wieseke et al. (2012) & $\begin{array}{l}\text { Trope \& Liberman (2003); } \\
\text { Henderrson (2009) }\end{array}$ \\
\hline $\begin{array}{l}\text { Contemporary interpretivist } \\
\text { identity theory }\end{array}$ & $\begin{array}{l}\text { O’Brien } \quad \& \quad \text { Linehan } \\
(2019)\end{array}$ & $\begin{array}{lcr}\text { Alvesson } & \text { et al. (2008); } \\
\text { Alvesson } & \text { (2010); Ramarajan } \\
(2014) & & \\
\end{array}$ \\
\hline
\end{tabular}




\begin{tabular}{|c|c|c|}
\hline Corporate professionalization & \begin{tabular}{|l|} 
Wylie, Sturdy \& Wright \\
$(2014)$
\end{tabular} & Muzio et al. (2011) \\
\hline Emotional contagion theory & Wieseke et al. (2012) & Barsade (2002) \\
\hline $\begin{array}{l}\text { Entity theory \& Incremental } \\
\text { theory }\end{array}$ & Dries (2013) & Heslin et al. (2005) \\
\hline $\begin{array}{l}\text { Equity theory and social } \\
\text { comparison }\end{array}$ & Björkman et al. (2013) & Carrell \& Dittrich (1978) \\
\hline Exchange and equity theory & Blader \& Tyler (2009) & $\begin{array}{l}\text { Thibaut \& Kelley (1959); } \\
\text { Homans (1961); Blau (1964); } \\
\text { Adams (1965) }\end{array}$ \\
\hline Grounded theory & $\begin{array}{|lll|}\begin{array}{l}\text { O’Brien } \\
(2019)\end{array} & \& \quad \text { Linehan } \\
\end{array}$ & $\begin{array}{l}\text { Glaser and Strauss (1967); } \\
\text { Strauss and Corbin (1990) }\end{array}$ \\
\hline Identification theory & Horton et al. (2014) & Kreiner \& Ashforth (2004) \\
\hline Job embeddedness & Ng \& Feldman (2009) & Mitchell et al. (2001) \\
\hline $\begin{array}{ll}\begin{array}{l}\text { Leader } \\
\text { entrepreneurship }\end{array} & \text { identity } \\
\end{array}$ & Steffens et al. (2018) & $\begin{array}{l}\text { Reicher \& Hopkins (2003); } \\
\text { Reicher et al. (2005); Steffens } \\
\text { et al. (2014) }\end{array}$ \\
\hline Moral identity theory & Hu \& Jiang (2018) & $\begin{array}{l}\text { Aquino \& Reed (2002); } \\
\text { Weaver (2006) }\end{array}$ \\
\hline Organizational identity theory & Walsh \& Gordon (2008) & Dutton et al. (1994) \\
\hline Organizational support theory & Björkman et al. (2013) & $\begin{array}{l}\text { Eisenberger, Fasolo, \& Davis- } \\
\text { LaMastro (1990) }\end{array}$ \\
\hline Signaling tl & Tsai & \\
\hline \multirow{7}{*}{$\begin{array}{l}\text { Social categorization \& Self- } \\
\text { categorization theory }\end{array}$} & allier (2010) & $\begin{array}{lll}\text { Turner (1985); } & \text { Turner and } \\
\text { Oakes (1997) } & & \\
\end{array}$ \\
\hline & Blader \& Tyler (2009) & Turner (1985) \\
\hline & $\begin{array}{|lll|}\begin{array}{l}\text { Hallier } \\
(2011)\end{array} & \& & \text { Summers } \\
\end{array}$ & Turner (1985) \\
\hline & \begin{tabular}{|l|} 
Héliot et al. (2020) \\
\end{tabular} & Turner et al. (1987) \\
\hline & Horton et al. (2014) & \begin{tabular}{|lll} 
Corley (2004); Magee \& \\
Galinsky (2008)
\end{tabular} \\
\hline & Steffens et al. (2018) & $\begin{array}{l}\text { Hogg \& Terry (2000); Haslam } \\
\text { (2001); van Dick (2001); } \\
\text { Ashfort, Harrison, \& Corley } \\
\text { (2008); Blader \& Tyler (2009) }\end{array}$ \\
\hline & Wieseke et al. (2012) & Turner et al. (1987) \\
\hline \multirow{2}{*}{ Social exchange theory } & Björkman et al. (2013) & Cropanzano \& Mitchell (2005) \\
\hline & Hu \& Jiang (2018) & Blau (1964) \\
\hline \multirow{5}{*}{ Social identity theory } & Baldry \& Hallier (2010) & $\begin{array}{l}\text { Mackie et al. (1990); Oakes et } \\
\text { al. (1991); McGarty et al. } \\
(1993,1994) ; \text { Balaam and } \\
\text { Haslam (1998) }\end{array}$ \\
\hline & Blader \& Tyler (2009) & $\begin{array}{l}\text { Tajfel \& Turner (1979); Tyler } \\
\text { \& Blader (2002; 2003) }\end{array}$ \\
\hline & Guan et al. (2016) & Tajfel \& Turner (1986) \\
\hline & $\begin{array}{|lll|}\begin{array}{l}\text { Hallier } \\
(2011)\end{array} & \& & \text { Summers } \\
\end{array}$ & Tajfel \& Turner (1979) \\
\hline & Héliot et al. (2020) & Tajfel \& Turner $(1979 ; 1985)$ \\
\hline
\end{tabular}




\begin{tabular}{|c|c|c|}
\hline & $\begin{array}{l}\text { Honeycutt \& Rosen B. } \\
(1997)\end{array}$ & Tajfel \& Turner (1985) \\
\hline & Horton et al. (2014) & Tajfel \& Turner (1979) \\
\hline & Steffens et al. (2018) & $\begin{array}{l}\text { Tajfel \& Turner (1979); Turner } \\
\text { et al. (1987); Ashforth \& Mael } \\
\text { (1989); Ellemers (2012) }\end{array}$ \\
\hline & \begin{tabular}{|l|} 
Topa \& Perez-Larrazabal \\
$(2016)$
\end{tabular} & Hogg (2005); Goldman (2006) \\
\hline & Tsai et al. (2015) & $\begin{array}{l}\text { Ashforth \& Mael (1989); } \\
\text { Turban et al. (2001) }\end{array}$ \\
\hline & Walsh \& Gordon (2008) & Turner (1982) \\
\hline & Wieseke et al. (2012) & $\begin{array}{l}\text { Tajfel \& Turner (1979); Hogg } \\
\text { \& Abrams (1988) }\end{array}$ \\
\hline Social learning theory & \begin{tabular}{|l|} 
Topa \& Perez-Larrazabal \\
$(2016)$
\end{tabular} & Bandura (1977) \\
\hline Theory of leader development & Chan \& Drasgow (2001) & \\
\hline Trait and control models & Pohler \& Willness (2014) & $\begin{array}{l}\text { Legge \& Exley (1975); Preece } \\
\text { \& Nicol (1980) }\end{array}$ \\
\hline Trait-activation theory & Guan et al. (2016) & Tett \& Burnett (2003) \\
\hline Trichotomy of Needs Theory & Chen (2008) & Harrell \& Stahl (1984) \\
\hline Uncertain identity theory & Wieseke et al. (2012) & $\operatorname{Hogg}(2000)$ \\
\hline
\end{tabular}

\section{2 - Main findings of extracted articles and further research suggestions}

Performing the content analysis, this study gathers in Tables 4 and 5 the main findings of each article and the suggestions for further research proposed by the authors.

Figure 7 - Methods of data gathering employed in extracted articles

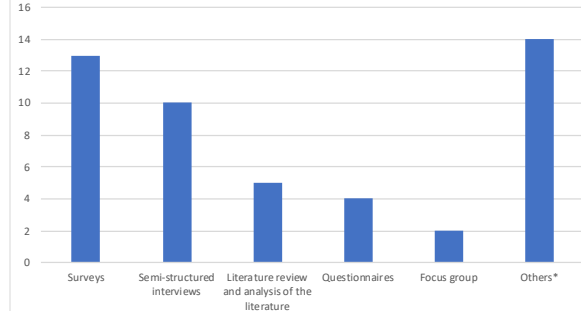

Figure 8 - Types statistical units considered in selected articles

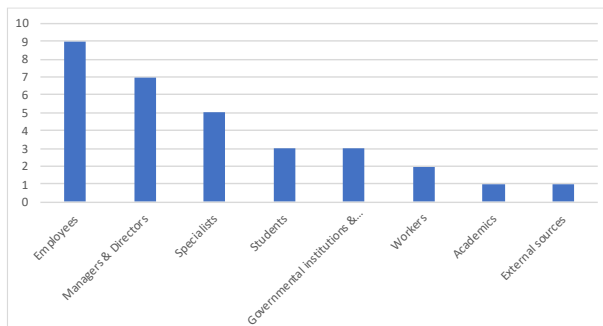

* Direct observation, Self-report, Cross-site analysis, Case-study, Items from archival data, Diary study, Public sources of data, Analysis of media reports of contemporary developments, Memory-work, Intersectional analysis, Observation and participation in meetings, Frequent informal discussion, Documents, Interviews (generic). 
Figure 9 - Statistical units provenience

Table 4 - Main findings of extracted articles

\begin{tabular}{|c|c|}
\hline Article's authors & Main findings \\
\hline Katz (1978) & $\begin{array}{l}\text { Job and organizational longevity affect the relationship between } \\
\text { job satisfaction and the five tasks dimension: skill variety, task } \\
\text { identity, task significance, autonomy, and feedback-from-job. }\end{array}$ \\
\hline $\begin{array}{l}\text { Honeycutt \& Rosen } \\
\text { (1997) }\end{array}$ & $\begin{array}{l}\text { Salient identity influences the individual career decision. Flexible } \\
\text { career policies attract all identity categories, but they diverge in } \\
\text { the level of attraction for traditional or dual career paths. }\end{array}$ \\
\hline Alvesson (2001) & $\begin{array}{l}\text { The role of knowledge in knowledge-intensive organizations is } \\
\text { ambiguous. } \\
\text { Knowledge-intensive workers and organizations could } \\
\text { experiment with instability, arbitrariness, and vulnerability. }\end{array}$ \\
\hline Chan \& Drasgow (2001) & $\begin{array}{l}\text { Personality, values, and past leadership experience are related to } \\
\text { the motivation to lead through leadership self-efficacy. The } \\
\text { general cognitive ability is unrelated to motivation to lead. } \\
\text { Motivation to lead assessed at the point of organizational entry is } \\
\text { at least predictive of subsequent behavioral ratings of leadership } \\
\text { potential. }\end{array}$ \\
\hline $\begin{array}{l}\text { Darr \& Scarselletta } \\
(2002)\end{array}$ & $\begin{array}{l}\text { The professional identity of technicians depends on their } \\
\text { interactions with clients and professional collaborators. }\end{array}$ \\
\hline Caldwell (2003) & $\begin{array}{l}\text { The HR function is rarely able to - and/or the HR specialists are } \\
\text { rarely willing to - reinvent its position in the organizations. }\end{array}$ \\
\hline $\begin{array}{l}\text { Farndale \& Brewster } \\
(2005)\end{array}$ & $\begin{array}{l}\text { Professional associations support the legitimation of HR identity, } \\
\text { but they cannot be considered the occupation controller. The } \\
\text { enrollments to HR management studies are diffused, showing a } \\
\text { substantial legitimation of the HR profession, but the certification } \\
\text { is not compulsory to work in an HR function. }\end{array}$ \\
\hline Roberts et al. (2005) & $\begin{array}{l}\text { The paper adds a means for human resource management: } \\
\text { "creating contexts that maximize the possibility for employees to } \\
\text { envision and enact their best-self." }\end{array}$ \\
\hline Chen (2008) & $\begin{array}{l}\text { IS personnel's job satisfaction is (1) independent from motivation } \\
\text { achievement; (2) positively related to job characteristics, } \\
\text { feedback, professionalism, and autonomy. }\end{array}$ \\
\hline Walsh \& Gordon (2008) & $\begin{array}{l}\text { Individuals' professional identity can be created through } \\
\text { organizational identification based on the improvement of } \\
\text { distinction and status obtained by individuals from the } \\
\text { membership in professional groups. }\end{array}$ \\
\hline
\end{tabular}




\begin{tabular}{|c|c|}
\hline Wright (2008) & $\begin{array}{l}\text { Human resources management's professional identity is not } \\
\text { directly affected by the achievement of greater self-awareness and } \\
\text { status originated by acquiring a role as a business partner / internal } \\
\text { consultant. }\end{array}$ \\
\hline Blader \& Tyler (2009) & $\begin{array}{l}\text { (1) Employees' social identity has strong relations with their } \\
\text { extra-role behaviors. (2) Social identity explains the impact of } \\
\text { experimented procedural justice and economic outcomes resulting } \\
\text { from group membership. (3) The motivational power contributes } \\
\text { to the employees' social identity development. }\end{array}$ \\
\hline Ng \& Feldman (2009) & $\begin{array}{l}\text { Job embeddedness contributes to greater performance, greater } \\
\text { creativity, and less counterproductive behaviors at work. }\end{array}$ \\
\hline Baldry \& Halier (2010) & $\begin{array}{l}\text { Reducing the emotional autonomy of personnel can divide } \\
\text { employees' identity and the identity of the organization. The } \\
\text { employees show the identity the management expects to see, } \\
\text { hiding their creative identity. }\end{array}$ \\
\hline $\begin{array}{l}\text { Hallier } \\
(2011)\end{array} \quad \& \quad$ Summers & $\begin{array}{l}\text { Human resources management students rarely change their } \\
\text { professional goals concerning participation in the human } \\
\text { resources profession. Nevertheless, they reach a greater awareness } \\
\text { of what "doing HRM" means adapting their idea to professional } \\
\text { expectations and learning experiences. }\end{array}$ \\
\hline $\begin{array}{l}\text { Pritchard \& Symon } \\
(2011)\end{array}$ & $\begin{array}{l}\text { The re-orientation of the strategic role of HR has consequences } \\
\text { that challenge the results in the literature. In fact, after this re- } \\
\text { orientation, HR managers tried to restore their professional } \\
\text { identity, underlining their previous role in welfare, perceived as } \\
\text { vacant after this change. }\end{array}$ \\
\hline Roche \& Teague (2012) & $\begin{array}{l}\text { HR managers are acquiring new strategic competencies. } \\
\text { Therefore, their participation in the definition of firms' strategy } \\
\text { has to go beyond the simple reaction to retrenchment measures. }\end{array}$ \\
\hline Wieseke et al. (2012) & $\begin{array}{l}\text { The results show that the corporate headquarters' physical } \\
\text { distance enhances sales representatives' work team identification } \\
\text { and diminishes their organizational identification. }\end{array}$ \\
\hline Björkman et al. (2013) & $\begin{array}{l}\text { People who have been identified as talented have more probability } \\
\text { of developing positive attitudes and competencies at work. } \\
\text { Therefore, informing talent individuals to have been identified as } \\
\text { talent improve their motivation. }\end{array}$ \\
\hline Dries (2013) & $\begin{array}{l}\text { The authors found discrepancies, theoretical perspectives, } \\
\text { tensions, and assumptions in talent management literature. }\end{array}$ \\
\hline $\begin{array}{l}\text { Horton, McClelland, } \\
\text { Griffin (2014) }\end{array}$ & $\begin{array}{l}\text { Organizational identification is connected to the different } \\
\text { hierarchical levels fulfilled by employees. Operational, middle- } \\
\text { level, and strategic level personnel identify with career and } \\
\text { professional groups, careers, and organizations. }\end{array}$ \\
\hline $\begin{array}{lll}\text { Pohler } & \& & \text { Willness } \\
(2014) & & \end{array}$ & $\begin{array}{l}\text { The philosophy of HR strategy has to be clarified. A more } \\
\text { strategic role of HR management could result in a decrease in } \\
\text { stakeholders' recognition. It could hinder professionalization by } \\
\text { reducing external legitimacy. }\end{array}$ \\
\hline $\begin{array}{l}\text { Wylie, Sturdy, Wright } \\
(2014)\end{array}$ & $\begin{array}{l}\text { (1) Credibility is directly connected to perceived expertise, which } \\
\text { is relative and domain-oriented. It can be affected by the } \\
\text { occupational background. (2) The expert status is fragile, and the } \\
\text { changing nature of management functions influences it. (3) HR } \\
\text { has to convince others about the value of its activities that are }\end{array}$ \\
\hline
\end{tabular}




\begin{tabular}{|c|c|}
\hline & $\begin{array}{l}\text { performed in the bottom line. (4) Credibility is fundamental for } \\
\text { facing the ambiguity problem in the HR function. }\end{array}$ \\
\hline Guan et al. (2015) & $\begin{array}{l}\text { Professional identity, career variety, and organizational support } \\
\text { are predictors for the strategic competence of HR managers. This } \\
\text { relation is mediated by career adaptation. }\end{array}$ \\
\hline Pritchard \& Fear (2015) & $\begin{array}{l}\text { The resignation of experts belonging to } \mathrm{T} \& \mathrm{D} \text { groups can } \\
\text { compromise the credibility of that group, threatening its identity. }\end{array}$ \\
\hline Tsai et al. (2015) & $\begin{array}{l}\text { Perceived corporate ability, positive publicity, and CSR are } \\
\text { directly related to pursuit intention and indirectly related to word- } \\
\text { of-mouth. Career expectations mediate these relationships. }\end{array}$ \\
\hline $\begin{array}{l}\text { Topa \& } \\
\text { Perez-Larrazabal (2016) }\end{array}$ & $\begin{array}{l}\text { Negative and inadequate mentoring may increase co-workers' } \\
\text { undermining and negatively influence newcomers' learning, } \\
\text { reducing their embeddedness. If the level of group identity is high, } \\
\text { this effect declines. }\end{array}$ \\
\hline $\begin{array}{l}\text { Mooney, Harris \& } \\
\text { Ryan (2016) }\end{array}$ & $\begin{array}{l}\text { (1) A necessary condition for gaining the respect of other peers is } \\
\text { the devotion to the job and hospitality passion. (2) Professional } \\
\text { identity is directly connected to co-workers and customer esteem. } \\
\text { This moderates the negative view of hospitality careers. (3) The } \\
\text { perception of control and variety enhance the longevity of careers. } \\
\text { (4) The longevity of a career is directly connected to a positive } \\
\text { workplace and the well-being of workers. (5) Four career enablers } \\
\text { that help career progression: gaining qualifications; undergoing } \\
\text { training and development; receiving the backing from influential } \\
\text { mentors; demonstrating flexibility. (6) Social competencies } \\
\text { facilitate access to developmental opportunities. }\end{array}$ \\
\hline Mackay (2017) & $\begin{array}{l}\text { Knowledge capabilities are expanded by continuous development, } \\
\text { maintaining the coherence of vocational identity. }\end{array}$ \\
\hline Steffens et al. (2017) & $\begin{array}{l}\text { Employees' perception of leaders' identity entrepreneurship } \\
\text { increases work engagement and decrease burnout and turnover } \\
\text { intentions. }\end{array}$ \\
\hline Hu \& Jiang (2018) & $\begin{array}{l}\text { Employees' morale identity and personal and familiar needs } \\
\text { should be integrated into HR policies to promote employees' } \\
\text { voices. }\end{array}$ \\
\hline \begin{tabular}{|l|} 
Currie, Spyridonidis \& \\
Oborn (2019) \\
\end{tabular} & $\begin{array}{l}\text { Knowledge sharing of employees depends on their perception of } \\
\text { HR policies' effect on their identity and legitimacy. }\end{array}$ \\
\hline Héliot et al. (2019) & $\begin{array}{l}\text { Professional and religious identity are connected through the } \\
\text { following variables: personal preferences, the fit between } \\
\text { religious identity and job-related concerns, and the organization's } \\
\text { policies, practices, and expectations. }\end{array}$ \\
\hline Pettit \& Crossan (2019) & $\begin{array}{l}\text { (1) There is a symbiotic relationship between the organization, } \\
\text { occupational members, and product created. (2) Occupational } \\
\text { members both facilitate and disrupt strategic renewal in } \\
\text { organizations. (3) Non-managerial actors are fundamental in } \\
\text { doing strategies. (4) Affirming is critical during collaborative } \\
\text { work. }\end{array}$ \\
\hline $\begin{array}{l}\text { O’Brien \& Linehan } \\
(2019)\end{array}$ & $\begin{array}{l}\text { People that are experimenting with emotional dissonance face } \\
\text { conflict emotions because of the competition between selves and } \\
\text { values. Their success is not to be themselves, but to identify what } \\
\text { self needs to be salient in a certain situation. }\end{array}$ \\
\hline
\end{tabular}


Table 5-Opportunities for further research

\begin{tabular}{|c|c|}
\hline Article & Proposals for further researches \\
\hline Katz (1978) & $\begin{array}{l}\text { (1) Longitudinal studies for understanding if an inverse relationship } \\
\text { between professional satisfaction and job position exists. (2) } \\
\text { Understanding the meaning of "new job". (3) Understanding individual } \\
\text { and occupational differences in job longevity context: role of possible } \\
\text { moderators (e.g. higher-order need strength, tolerance for uncertainty, } \\
\text { and need for achievement and affiliation). }\end{array}$ \\
\hline $\begin{array}{l}\text { Honeycutt \& Rosen } \\
\text { (1997) }\end{array}$ & $\begin{array}{l}\text { (1) Is there a minimum salary limit below which it becomes important? } \\
\text { (2) Do men with salient family identities take more time to fulfill their } \\
\text { family obligations? }\end{array}$ \\
\hline $\begin{array}{l}\text { Chan \& Drasgow } \\
(2001)\end{array}$ & $\begin{array}{l}\text { (1) Are extroversion and sociability general antecedents for self- } \\
\text { efficacy leadership? (2) Do people who are open to experience and more } \\
\text { extroverted have more leadership experiences in the past? (3) Verify } \\
\text { that general cognitive ability is not an antecedent of motivation to lead } \\
\text { with large samples. (4) Study of the empirical relationships between } \\
\text { individual-differences constructs and ultimate performance criteria to } \\
\text { find theoretical explanations for connections. (5) Call for more } \\
\text { empirical papers on motivation to lead. }\end{array}$ \\
\hline $\begin{array}{l}\text { Farndale } \& \\
\text { Brewster (2005) }\end{array}$ & $\begin{array}{l}\text { (1) Empirical works about the difference between certified and non- } \\
\text { certified HR managers' effects in their respective workplaces. (2) } \\
\text { Meaning of professionalism in HR. }\end{array}$ \\
\hline $\begin{array}{l}\text { Roberts et al. } \\
(2005)\end{array}$ & $\begin{array}{l}\text { (1) Study of mechanism that enhances or reduces the probability of } \\
\text { positive change in Reflected Best-Self Portrait. (2) Examining the } \\
\text { quality of the relationship between employees and feedback givers. (3) } \\
\text { Study the discrepancy between employees' own image and others' } \\
\text { reflections. (4) Examining the influence of macro-social context on } \\
\text { Reflected Best-Self Portrait. }\end{array}$ \\
\hline Chen (2008) & $\begin{array}{l}\text { (1) Integrating more variable for studying job satisfaction: job } \\
\text { performance, job behavior, job attitude. (2) Organizational factors } \\
\text { should be included in a job satisfaction study. }\end{array}$ \\
\hline $\begin{array}{l}\text { Walsh \& Gordon } \\
(2008)\end{array}$ & $\begin{array}{l}\text { Call for more empirical papers on the creation of the individual work } \\
\text { identity. }\end{array}$ \\
\hline $\begin{array}{l}\text { Blader } \& \quad \text { Tyler } \\
(2009)\end{array}$ & $\begin{array}{l}\text { (1) Alternative research methods to understand causal relations between } \\
\text { social identity and employees' extra-role behaviors. (2) Explore respect } \\
\text { as an important element of social identity and (intragroup) relational } \\
\text { identity. (3) Study the relations between social identity and other } \\
\text { variables (e.g., affective commitment). }\end{array}$ \\
\hline $\begin{array}{l}\mathrm{Ng} \& \text { Feldman } \\
(2009)\end{array}$ & $\begin{array}{l}\text { (1) Examination of relations between embeddedness, performance, and } \\
\text { turnover. (2) Relations between embeddedness and the dimensions of } \\
\text { performance. (3) Investigating overlapping conditions of leaving an } \\
\text { organization and leaving an occupation. }\end{array}$ \\
\hline $\begin{array}{l}\text { Hallier \& Summers } \\
(2011)\end{array}$ & $\begin{array}{l}\text { Study the outcomes of vocational identity in other nascent professions } \\
\text { [other than human resources]. }\end{array}$ \\
\hline $\begin{array}{l}\text { Pritchard \& Symon } \\
(2011)\end{array}$ & $\begin{array}{l}\text { The re-orientation of the strategic role of HR has consequences that } \\
\text { challenge the results in the literature. In fact, after this re-orientation, } \\
\text { HR managers tried to restore their professional identity, underlining } \\
\text { their previous role in welfare, perceived as vacant after this change. }\end{array}$ \\
\hline
\end{tabular}




\begin{tabular}{|c|c|}
\hline $\begin{array}{l}\text { Wieseke et al. } \\
(2012)\end{array}$ & $\begin{array}{l}\text { (1) Examining the cross-industry stability, decreasing organization } \\
\text { identification of salespeople who work far from headquarters. (2) } \\
\text { Investigate the effect of positive stereotypes in marketing and } \\
\text { management. }\end{array}$ \\
\hline $\begin{array}{l}\text { Björkman et al. } \\
(2013)\end{array}$ & $\begin{array}{l}\text { (1) Longitudinal studies to understand if the awareness of being } \\
\text { identified as talented could encourage better alternatives in the labor } \\
\text { market. (2) Examine the effect of cultural and institutional factors on } \\
\text { the turnover of employees. (3) Comparative and qualitative analysis for } \\
\text { the effect of supervisors' communication on talented people's talent } \\
\text { status. }\end{array}$ \\
\hline Dries (2013) & $\begin{array}{l}\text { The authors have collected several further research suggestions in Table } \\
4 \text { of their paper. } \\
\text { Call for more empirical papers. }\end{array}$ \\
\hline Horton et al. (2014) & $\begin{array}{l}\text { (1) Longitudinal analysis to understand if workplace identification } \\
\text { changes may be triggered by key changes in an individual's career. (2) } \\
\text { Longitudinal analysis to understand if group norms and values can be } \\
\text { expected to be socially constructed, while group members develop an } \\
\text { understanding of what is expected and appropriate at different } \\
\text { hierarchical levels. (3) How the mechanisms through which } \\
\text { identification processes are built and evolve? }\end{array}$ \\
\hline $\begin{array}{l}\text { Pohler \& Willness } \\
\text { (2014) }\end{array}$ & $\begin{array}{l}\text { Investigate how the HR function's potentially conflictual role, who has } \\
\text { to balance organization and personnel interests, is faced by HR } \\
\text { managers. }\end{array}$ \\
\hline $\begin{array}{l}\text { Wylie, Sturdy \& } \\
\text { Wright (2014) }\end{array}$ & $\begin{array}{l}\text { (1) Comparison between the role of change agents in how HR functions } \\
\text { has a different status. (2) Study the role of change agents in a more } \\
\text { consolidated HR field (e.g., centers of excellence). }\end{array}$ \\
\hline Tsai et al. (2015) & $\begin{array}{l}\text { Concerning the candidate's occupation preferences, verify the role of } \\
\text { other factors: candidates' cultural preferences, subjective adaptation, } \\
\text { network comfort, neuroticism, extroversion, pleasantness, opennes, } \\
\text { awareness. }\end{array}$ \\
\hline $\begin{array}{l}\text { Mooney, Harris \& } \\
\text { Ryan (2016) }\end{array}$ & $\begin{array}{l}\text { (1) Quantitative test of variables influencing career progressions. (2) } \\
\text { Qualitative and quantitative research on the effect of age, gender, and } \\
\text { ethnicity on career longevity. }\end{array}$ \\
\hline $\begin{array}{l}\text { Topa \& Perez- } \\
\text { Larrazabal (2016) }\end{array}$ & $\begin{array}{l}\text { Evaluate the use of more objective measures for strengthening the } \\
\text { relationship between negative mentoring and co-worker undermining } \\
\text { (also considering international contexts). }\end{array}$ \\
\hline $\begin{array}{l}\text { Steffens et al., } \\
(2017)\end{array}$ & $\begin{array}{l}\text { (1) Extension of the work to understand other moderators' position of } \\
\text { the leader's identity entrepreneurship. (2) Clarify if there is a dark side } \\
\text { of identity entrepreneurship: less beneficial effects on employee health. } \\
\text { (3) How does the composition of multiple workgroups relate to burnout, } \\
\text { employee engagement, and turnover? }\end{array}$ \\
\hline Mackay (2017) & $\begin{array}{l}\text { (1) How do other professions look at continuous development? (2) } \\
\text { Specific claims of the profession in aspiring to professional status. (3) } \\
\text { How do established professions theorize professional learning? (4) } \\
\text { Does compulsory CPD limit understanding of professional } \\
\text { development? }\end{array}$ \\
\hline Hu \& Jiang (2018) & $\begin{array}{l}\text { (1) Examine differential effects of employee-oriented HR management } \\
\text { on the promoting and prohibitive speech behavior. (2) Ask colleagues } \\
\text { and supervisors of respondents to evaluate vocal behaviors: use multiple }\end{array}$ \\
\hline
\end{tabular}




\begin{tabular}{|l|l|}
\hline & $\begin{array}{l}\text { raters to have multiple voices. (3) Definition and use of a complete scale } \\
\text { of variables to acquire an exhaustive picture of trust and voice. }\end{array}$ \\
\hline Currie et al. (2019) & $\begin{array}{l}\text { Increase contexts for evaluating the interaction of HR practices and } \\
\text { organizations. }\end{array}$ \\
\hline Guan et al. (2015) & $\begin{array}{l}\text { (1) Examine the specific roles of these dimensions [vocational identity, } \\
\text { organizational support, career variety] in developing HR managers' } \\
\text { strategic skills. (2) HR experience variables to explain the effect of } \\
\text { vocational identity. (3) Experimental and longitudinal designs to } \\
\text { understand mutual effects of vocational identity, career variety and } \\
\text { organizational support, career adaptability, and strategic skills. }\end{array}$ \\
\hline $\begin{array}{l}\text { O'Brien \& Linehan } \\
(2019)\end{array}$ & $\begin{array}{l}\text { (1) Examination about building multiple selves and the practices that } \\
\text { help or hind this process. (2) Analysis of the influence of specific } \\
\text { identity domains on dissonance. }\end{array}$ \\
\hline $\begin{array}{l}\text { Pettit \& Crossan } \\
(2019)\end{array}$ & $\begin{array}{l}\text { (1) Examine the distinction between tensions and alert on organizational } \\
\text { risks. (2) Analyzing the long-term consequences of strategic renewal } \\
\text { for organizations and occupations. (3) Analyzing the role of timing and } \\
\text { models of occupational identity for future strategic initiatives. }\end{array}$ \\
\hline Hèliot et al. (2020) & $\begin{array}{l}\text { (1) Examine the effects of diversity policies on individuals, groups and } \\
\text { organizations with different religious identities. (2) Examine the effects } \\
\text { of faults in activating religious identity compared to other forms of } \\
\text { identity in the workplace. (3) Examine the forms of workplace climate } \\
\text { and related HR policies and practices to activate or minimize fault lines } \\
\text { derived from religious identity. }\end{array}$ \\
\hline
\end{tabular}

\section{3 - Analysis of emerging results from extracted articles}

In this paragraph, the results emerging from the content analysis of the selected articles are considered. In particular, the paragraph is focused on the research questions RQ1 and RQ2. The study examines the various thematic areas that emerged during the in-depth inspection of the articles' content extracted from the existing literature, highlighting the various authors' considerations, conflicts, and crises.

\subsection{1 - Motivation, satisfaction, and well-being of employees}

Employees' satisfaction is firmly linked to their experiences in organizations, their professional and private well-being, and the harmony between their internal and external values, which enhance the expression of the authentic self (Hèliot et al., 2020). Their well-being is strongly connected to their organizational identification and leaders' actions. It can limit the onset of complex pathologies as stress, anxiety, and depression (Horton et al., 2014; Steffens et al., 2017) that have physical and psychological consequences.

One of the antecedents of satisfaction is motivation, which is positively related to firms' versatility (Katz, 1978), ability to valorize talents (Björkman et al., 2013), employees' perceived degree of professional and emotional autonomy (Baldry \& Hallier, 2010; Chen, 2008), and opportunities to influence their organizations (Katz, 1978). The more these variables are 
developed, the more passion beyond commitment will be developed by employees (Dries, 2013), who will achieve professional identification fundamental for productivity and personal growth (Guan et al. 2015). Obviously, job longevity relies on developing such requisites (Katz, 1978; Mooney et al., 2016).

Ensuring the motivation is complex because of the needs connected to organizational identity change according to employees' hierarchical positions (Katz, 1978). Moreover, firms' normative nature impedes the complete support of every employee's specific needs (Dries, 2013).

\subsection{2 - The "crisis" of HR management}

In the examined literature, there are several pieces of evidence about the status of "crisis" that HR has been experimenting in the last two decades. In particular, the literature asks if HR function has the necessary competencies to achieve a professional legitimation (Farndale \& Brewster, 2005; Guan et al., 2015), that is widely desired by HR managers (Mackay, 2017) despite some resistance of other firms' departments, e.g., accountancy and finance (Wright, 2008). HR is sometimes defined as a semi-profession (Mackay, 2017; Wright, 2008), and the strategic renewal of the profession is seen as rather improbable (Caldwell, 2003; Pohler \& Willness, 2014; Pritchard \& Symon, 2011). Although the professional associations are growing in their number, formal rules for legitimizing the HR profession still do not exist.

A dual perspective characterizes the HR function. On the one hand, there is the idea of HR as an administrative partner devoted to adaptive interventions following organizational and labor market crises (Guan et al., 2015). On the other hand, the HR function is seen as a possible future strategic partner, despite positions that underline this function's weakening due to this new interpretation (Roche \& Teague, 2012).

The HR function is experimenting with a contradictory position. In fact, it has to support the employees' needs and, at the same time, coordinate the management processes (Mackay, 2017). For this reason, the legitimation of the HR profession is critical for improving its credibility (Wylie et al., 2014) and to configure HR function as real strategic support for the firms (Guan et al., 2015). To achieve this strategic renewal, the involvement of nonmanagerial personnel is fundamental (Pettit \& Crossan, 2019), as well as the experience of human resources (Al-Raggad, 2014). Therefore, the HR professional legitimation will be achieved throughout the HR function's involvement at the strategic decision table. For this reason, HR managers have to adequate and develop their strategic knowledge. 


\subsection{3 - Identity in workplace}

People build their identity also through their workgroups. The achievement of identity, i.e., self-awareness in their environments (Hèliot et al., 2020), is performed through the participation in inclusive but distinctive groups (Darr \& Scarselletta, 2002; Horton et al., 2014) that allow to employees the perception of their identity as unique, special and ameliorative of their status (Hallier \& Summers, 2011; Walsh \& Gordon, 2008). In these groups, identity is modeled by the sense of belonging (Steffens et al., 2017), dynamically and evolutionarily (Mackay, 2017), to achieve the fundamental objective of organizational identification (Horton et al., 2014).

The foundations of this awareness's development are social identity, which is firmly connected to the motivational power of workgroups (Blader \& Tyler, 2009) and to professional identity that, unfortunately, is a precarious identity domain due to its strong dependency on the context (Alvesson, 2001). The maturity of employees' professional and organizational identity is linked to their ability to emerge their different identity domains according to the specific situation (O'Brien \& Linehan, 2019).

All this let emerge the fundamental role of the HR function, which has to ensure to employees the possibility to show their best-self (Roberts et al., 2005), to allow them to find congruence between their identity and the identity of their organizations (Walsh \& Gordon, 2008), achieving the job embeddedness ( $\mathrm{Ng} \&$ Feldman, 2009). The HR function has to consider that organizational identity is connected to employees' hierarchical positions: the more the personnel Is involved in strategic roles, the more their organizational identification (Horton et al., 2014). Given that such a condition is critical for the stability and the improvement of firms' performances, HR management could have a fundamental strategic role.

Organizational identification is difficult to establish and easy to weaken (Dries, 2013). It finds its antecedents in mentors (Topa \& PerezLarrazabal, 2016), colleagues, customers (Mooney et al., 2016), and sociofamiliar contexts. It is weakened by factors as, for example, the resignation of important group members (Pritchard \& Fear, 2015) or by the simple distance from the firm's headquarters (Wieseke et al., 2012). Moreover, globalization promotes the self-entrepreneurship and distances individuals from organizational identification. Therefore, the career policies, together with the consideration of employees' socio-familiar problems, have a critical role. Furthermore, profound values can moderate or mediate the formation of this identity. The congruence of employees' morale and religious values with their organizational duties influence the individuals' reactions to firms' policies (Currie et al., 2019; Hèliot et al., 2020; Hu \& Jiang, 2018).

The labor market is complex, unstable and unable to guarantee longterms contracts to employees. HR policies have to modify their nature to 
attract talents and increasing the probability of their organizational identification. Such strategies ensure employees' motivation, well-being, productivity and performance. Without these foundations, firms will be always more perceived by employees as means to be used for the satisfaction of their extra-professional needs.

\subsection{4 - Career adaptation}

Changes in the labor market due to globalization modify the nature of HR function that has to adequate to new needs of integrating its traditional competencies with strategic skills. The exploiting of previous professional experiences could represent the key to achieving this objective (Guan et al., 2015) and facilitating the adaptation. HR experiments three different pressures: from employees, organizations (Honeycutt \& Rosen, 1997), and general market conditions. For this reason, the adaptation of this function depends on professional and organizational identity.

The HR department should operate to achieve the adaptation of employees to their career and the adaptation of organization to employees' careers. The equilibrium between these two tensions is critical for the development of professional and organizational identity.

The adaptation of organizations to employees' careers is achieved by awareness of their priorities and personal conditions. Firms can plan separated career paths to embrace employees' personal needs (Honeycutt \& Rosen, 1997). At the same time, employees should consider taking paths of continuous learning, showing flexibility, and following important mentors (Mooney et al., 2016). Considering these factors can avoid a high level of dropout.

Therefore, career adaptation should not be imposed but achieved throughout the smoothing of the existing distance between employees' and organizations' identity. Knowledge sharing and transfer (Currie et al., 2019; Ikyanyon \& Ode, 2017; Pierotti et al., 2014), performance management systems (Mahapa, Dzimbiri, \& Maphosa, 2015), the opportunity for employees to perceive the uniqueness of their identity in organizations (Guan et al., 2015), as well as the perception of working in an organization which considers their personal needs (Honeycutt \& Rosen, 1997; Walsh \& Gordon, 2008), are all antecedents to achieve this process.

\section{Conclusion}

The analysis performed allows answering the research questions through the following considerations.

Concerning the RQ1, the literature analysis let emerge that employees' motivation, satisfaction, and well-being are firmly connected with the organizational and professional identification (Horton et al., 2014; Steffens et 
al., 2017). The productivity and the personal growth of individuals are strongly linked to professional identity (Guan et al. 2015), which is enhanced by identifying individuals with their organizations. Therefore, the coherence between individuals and their organizations' perceptions is a fundamental antecedent for workers' and firms' performances (Walsh \& Gordon, 2008). Employees have many different ways to identify with their organization, depending on their values and identity development. Such a condition is delicate and characterized by a precarious equilibrium (Dries, 2013). In fact, it strongly depends on the external conditions and context, being subjected to the influence of elements that could be internal or external to the job place. Mentors (Topa \& Perez-Larrazabal, 2016), co-workers, customers (Mooney et al., 2016), family, and social environment produce effects on this equilibrium. For these reasons, firms have to seriously consider employees' vocational identity, integrating their development in their strategies and career paths.

Concerning the RQ2, the emerged results during this study's analysis show a "crisis" of HR identity (Mackay, 2017; Wright, 2008), negatively affecting employees' professional identity development. In particular, with the rapid evolution of information theory, the position of the HR department, often perceived as simple offices devoted to administrative tasks connected to labor, in many cases seems not to find a justification for its existence in the future (Mackay, 2017; Wright, 2008). Therefore, the HR function is experimenting with a "crisis" status that risks involving its identity and workers' professional identity.

In this sense, the flexibility of HR policies, leadership abilities, talent management, and appreciation of qualitative differences between employees' groups should constitute the fundamental principle of motivation and work attraction.

In conclusion, the literature highlights that career adaptation and organizational identification are increasingly dependent on firms' strategy and career-related policies that should always involve more HR functions. Therefore, the critical issues that involve the HR department's role could be overcome by a renovated role that it can assume considering the emerging needs, previously explained, concerning the valorization of qualitative differences between employees' groups.

What emerged from the answers to RQ1 and RQ2 finds its natural outcome in the analysis conducted for responding to RQ3. In particular, a synthesis is provided by the SLR analysis performed to identify the intersection of the identity concept and HR function in the organizations. This study highlighted the importance of this theme and the existence of a profound connection between the concepts of professional identity and HR function, seen as mutually influential elements in a potentially virtuous circle. 
These reflections encourage further development of research towards in-depth examinations on connections between these two themes. In particular, analyzing the development of professional identity through a renovated strategic role of the HR function, not restricted to a mere bureaucratic and administrative partner but extended to a promoter and supporter of employees' vocational identity development, appears attractive.

\section{References:}

1. Alvesson, M. (2001). Knowledge work: Ambiguity, image and identity. Human relations, 54(7), 863-886.

2. Baldry, C. \&. (2010). Welcome to the house of fun: Work space and social identity. Economic and Industrial Democracy, 31(1), 150-172.

3. Björkman, I., Ehrnrooth, M., Mäkelä, K., Smale, A., \& Sumelius, J. (2013). Talent or not? Employee reactions to talent identification. Human Resource Management, 52(2), 195-214.

4. Blader, S. L., \& Tyler, T. R. (2009). Testing and extending the group engagement model: Linkages between social identity, procedural justice, economic outcomes, and extrarole behavior. Journal of applied psychology, 94(2), 445.

5. Caldwell, R. (2003). The changing roles of personnel managers: old ambiguities, new uncertainties. Journal of management Studies, 40(4), 983-1004.

6. Chan, K.-Y., \& Drasgow, F. (2001). Toward a theory of individual differences and leadership: Understanding the motivation to lead. Journal of Applied Psychology, 86(3), 481-498.

7. Chen, L.-H. (2008). Job satisfaction among information system (IS) personnel. Computers in Human Behavior, 24(1), 105-118.

8. Currie, G., Spyridonidis, D., \& Oborn, E. (2019). The influence of HR practices upon knowledge brokering in professional organizations for service improvement: Addressing professional legitimacy and identity in health care. Human Resource Management, In Press.

9. Darr, A., \& Scarselletta, M. (2002). Technicians, clients, and professional authority: Structured interactions and identity formation in technical work. New Technology, Work and Employment, 17(1), 6173.

10. Dries, N. (2013). The psychology of talent management: A review and research agenda. Human Resource Management Review, 23(4), 272285.

11. Farndale, E., \& Brewster, C. (2005). In search of legitimacy: Personnel management associations worldwide. Human Resource Management Journal, 15(3), 33-48. 
12. Guan, Y., Yang, W., Zhou, X., Tian, Z., \& Eves, A. (2016). Predicting Chinese human resource managers' strategic competence: Roles of identity, career variety, organizational support and career adaptability. Journal of Vocational Behavior, 92, 116-124.

13. Hallier, J., \& Summers, J. (2011). Dilemmas and outcomes of professional identity construction among students of human resource management. Human Resource Management Journal, 21(2), 204-219.

14. Héliot, Y., Gleibs, I. H., Coyle, A., Rousseau, D., \& Rojon, C. (2020). Religious identity in the workplace: A systematic review, research agenda, and practical implications. Human Resource Management, 59(2), 153-173.

15. Honeycutt, T., \& Rosen, B. (1997). Family friendly human resource policies, salary levels, and salient identity as predictors of organizational attraction. Journal of Vocational Behavior, 50(2), 271290.

16. Horton, K., McClelland, C., \& Griffin, M. (2014). Defined by our hierarchy? How hierarchical positions shape our identifications and well-being at work. Human Relations, 67(10), 1167-1188.

17. Hu, X., \& Jiang, Z. (2018). Employee-oriented HRM and voice behavior: a moderated mediation model of moral identity and trust in management. International Journal of Human Resource Management, 29(5), 746-771.

18. Katz, R. (1978). Job longevity as a situational factor in job satisfaction. Administrative science quarterly, 23(2), 204-223.

19. Mackay, M. (2017). Identity formation: professional development in practice strengthens a sense of self. Studies in Higher Education, 42(6), 1056-1070.

20. Mooney, S. K., Harris, C., \& Ryan, I. (2016). Long hospitality careers - a contradiction in terms? International Journal of Contemporary Hospitality Management, 28(11), 2589-2608.

21. Ng, T. W., \& Feldman, D. C. (2009). Occupational embeddedness and job performance. Journal of Organizational Behavior: The International Journal of Industrial, Occupational and Organizational Psychology and Behavior, 30(7), 863-891.

22. O'Brien, E., \& Linehan, C. (2019). Problematizing the authentic self in conceptualizations of emotional dissonance. Human Relations, 72(9), 1530-1556.

23. Pettit, K. L., \& Crossan, M. M. (2020). Strategic renewal: Beyond the functional resource role of occupational members. Strategic Management Journal, 41(6), 1112-1138. 
24. Pohler, D., \& Willness, C. (2014). Balancing interests in the search for occupational legitimacy: The HR professionalization project in Canada. Human Resource Management, 53(3), 467-488.

25. Pritchard, K., \& Symon, G. (2011). Identity on the line: Constructing professional identity in a HR call centre. Work, Employment and Society, 25(3), 434-450.

26. Pritchard K., F. W. (2015). Credibility lost: Attempting to reclaim an expert identity in an HR professional context. Human Resource Management Journal, 25(3), 348-363.

27. Roberts, L. M., Dutton, J. E., Spreitzer, G. M., Heaphy, E. D., \& Quinn, R. E. (2005). Composing the reflected best-self portrait: Building pathways for becoming extraordinary in work organizations. Academy of Management Review, 30(4), 712-736.

28. Roche, W., \& Teague, P. (2012). Business partners and working the pumps: Human resource managers in the recession. Human Relations, 65(10), 1333-1358.

29. Steffens, N., Yang, J., Jetten, J., Haslam, S., \& Lipponen, J. (2018). The unfolding impact of leader identity entrepreneurship on burnout, work engagement, and turnover intentions. Journal of Occupational Health Psychology, 23(3), 373-387.

30. Topa, G., \& Perez-Larrazabal, J. (2016). Newcomers' learning and coworker undermining: moderated mediation analysis. Journal of Managerial Psychology, 31(5), 914-929.

31. Tsai, Y.-H., Lin, C.-P., Ma, H.-C., \& Wang, R.-T. (2015). Modeling corporate social performance and job pursuit intention: Forecasting the job change of professionals in technology industry. Technological Forecasting and Social Change, 99, 14-21.

32. Walsh, K., \& Gordon, J. (2008). Creating an individual work identity. Human Resource Management Review, 18(1), 46-61.

33. Wieseke, J., Kraus, F., Ahearne, M., \& Mikolon, S. (2012). Multiple identification foci and their countervailing effects on salespeople's negative headquarters stereotypes. Journal of Marketing, 76(3), 1-20.

34. Wright, C. (2008). Reinventing human resource management: Business partners, internal consultants and the limits to professionalization. Human Relations, 61(8), 1063-1086.

35. Wylie, N., Sturdy, A., \& Wright, C. (2014). Change agency in occupational context: Lessons for HRM. Human Resource Management Journal, 24(1), 95-110. 


\section{Other References:}

36. Adams, J. S. (1965). Inequity in social exchange. In L. Berkowitz, Advances in experimental social psychology (p. 267-299). New York: Academic Press.

37. Al-Raggad, M. A. (2014). Strategic human resource management and firm performance in Jordan listed firms. European scientific journal, 10(13).

38. Alvesson, M. (2010). Self-doubters, strugglers, storytellers, surfers and others: Images of self-identities in organization studies. Human Relations, 63(2), 193-217.

39. Alvesson, M., Lee Ashcraft, K., \& Thomas, R. (2008). Identity matters: Reflections on the construction of identity scholarship in organization studies. Organization, 15(1), 5-28.

40. Aquino, K., \& Reed, A. (2002). The self-importance of moral identity. Journal of Personality and Social Psychology, 83, 1423-1440.

41. Armishaw, D. D. (2007). Vocational Identity: It's about Working at Becoming. College Quarterly, 10(2), 1-13.

42. Aronson, E., Wilson, T. D., \& Akert, R. M. (1999). Social psychology (2nd ed.). New York: Longman.

43. Ashforth, B. E., \& Mael, F. (1989). Social identity theory and the organization. Academy of management review, 14(1), 20-39.

44. Balaam, B., \& Haslam, S. (1998). A Closer Look at the Role of Social Influence in the Development of Attitudes to Eating. Journal of Community and Applied Social Psychology, 8(3), 195-212.

45. Bandura, A. (1977). Self-efficacy: toward a unifying theory of behavioral change. Psychological Review, 84(2), 191-215.

46. Blau, P. M. (1964). Exchange and power in social life. New York, NY: Wiley.

47. Brown, A. D. (2015). Identities and identity work in organizations. International journal of management reviews, 17(1), 20-40.

48. Carrell, M. R., \& Dittrich, J. E. (1978). Equity theory: The recent literature, methodological considerations, and new directions. Academy of Management Review, 3(2), 202-210.

49. Corley, K. (2004). Defined by our strategy or our culture? Hierarchical differences in perceptions of organizational identity and change. Human Relations, 57(9), 1145-1177.

50. Cropanzano, R., \& Mitchell, M. S. (2005). Social ex- change theory: An interdisciplinary review. Journal of Management, 31, 874-900.

51. Danese, P., Manfè, V., \& Romano, P. (2018). A Systematic Literature Review on Recent Lean Research: State-of-the-art and Future Directions. International Journal of Management Reviews, 20(2), 579-605. 
52. de Zubielqui, C., Fryges, G., \& Jones, H. (2019). Social media, open innovation \& HRM: Implications for performance. Technological Forecasting and Social Change, 144(C), 334-347.

53. Denyer, D., \& Tranfield, D. (2009). Producing a systematic review. In D. A. Buchanan, \& A. Bryman, The Sage handbook of organizational research methods. London, UK: SAGE Publications.

54. Dutton, J. E., Dukerich, J. M., \& Harquail, C. V. (1994). Organizational images and member identification. Administrative Science Quarterly, 39, 239-263.

55. Eisenberger, R., Fasolo, P., \& Davis-LaMastro, V. (1990). Perceived organizational support and employee diligence, commitment, and innovation. Journal of Applied Psychology, 75(1), 51-59.

56. Ellemers, N. (2012). The group self. Science, 336, 848-852.

57. Erikson, E. H. (1963). Youth: Change and challenge. New York: Basic books.

58. Erikson, E. H. (1968). Identity: Youth and crisis. New York: Norton.

59. Erikson, E. H. (1982). The life cycle completed. New York: W.W. Norton \& Company.

60. Festinger, L. (1957). A theory of cognitive dissonance. Stanford: Stanford University Press.

61. Fink, A. (2013). Conducting Research Literature Reviews: From the Internet to Paper. London, UK: SAGE Pubblications.

62. Fredrickson, B. L. (2000). Cultivating positive emotions to optimize health and well-being. Prevention and treatment, 3(1).

63. Glaser, B. G., \& Strauss, A. L. (1967). The Discovery of Grounded Theory: Strategies for Qualitative Research. Chicago, Illinois: Aldine.

64. Goldman, A. (2006). High toxicity leadership: borderline personality disorder and the dysfunctional organization. Journal of Managerial Psychology, 21(8), 733- 746.

65. Grant, M. J., \& Booth, A. (2009). A typology of reviews: An analysis of 14 review types and associated methodologies. Health Information and Libraries Journal, 26(2), 91-108.

66. Grotevant, H. D. (1987). Toward a process model of identity formation. Journal of adolescent research, 2(3), 203-222.

67. Harrell, A. M., \& Stahl, M. J. (1984). McClelland's trichotomy of needs theory and the job satisfaction and work performance of CPA firm professionals. Accounting Organizations and Society, 9(3/4), 241-252.

68. Haslam, S. A. (2001). Psychology in organizations: The social identity approach (Rev. ed.). Thousand Oaks, CA: Sage Publications. 
69. Henry, P. (1993). Effectiveness of career-development courses for nontraditional premedical students: Improving professional identity. Psychological reports, 73(3), 915-920.

70. Heslin, P. A., Latham, G. P., \& Vandewalle, D. (2005). The effect of implicit person theory on performance appraisals. Journal of Applied Psychology, 90(5), 842-856.

71. Hogg, M. (2005). All animals are equal but some animals are more equal than others: social identity and marginal membership. In K. Williams, J. Forgas, \& W. von Hippel, The Social Outcast: Ostracism, Social Exclusion, Rejection, and Bullying (p. 243-261). New York: Psychology Press.

72. Hogg, M. A. (2000). Subjective Uncertainty Reduction Through SelfCategorization: A Motivational Theory of Social Identity Processes. European Review of Social Psychology, 11(1), 223-255.

73. Hogg, M. A., \& Abrams. (1988). Social identifications: A social psychology of intergroup relations and group processes. London: Routledge.

74. Hogg, M. A., \& Terry, D. I. (2000). Social identity and selfcategorization processes in organizational contexts. Academy of Management Review, 25, 121-140.

75. Homans, G. (1961). Social behavior: Its elementary forms. New York: Harcourt, Brace, World.

76. Ikyanyon, D. N., \& Ode, E. (2017). Fostering Knowledge Transfer through High- Performance HRM Practices: The Mediating Role of Affective Commitment. European Scientific Journal, ESJ, 13(13), 374.

77. Klotz, V. K., Billet, S., \& Winther, E. (2014). Promoting workforce excellence: formation and relevance of vocational identity for vocational educational training. Empirical Research in Vocational Education and Training, 6(1).

78. Kreiner, G. E., \& Ashforth, B. E. (2004). Evidence toward an expanded model of organizational identification. Journal of Organizational Behavior: The International Journal of Industrial, Occupational and Organizational Psychology and Behavior, 25(1), 127.

79. Legge, K., \& Exley, M. (1975). Authority, ambiguity and adaptation: The personnel specialist's dilemma. Industrial Relations Journal, 6, 51-65.

80. Mackie, D. M., Worth, L. T., \& Asuncion, A. G. (1990). Processing of Persuasive Ingroup Messages. Journal of Personality and Social Psychology, 58(5), 812-822. 
81. Magee, J., \& Galinsky, A. (2008). Social hierarchy: The selfreinforcing nature of power and status. Academy of Management Annals, 2(1), 351-398.

82. Mahapa, M., Dzimbiri, L., \& Maphosa, F. (2015). Performance management hurdles in a public health sector organisation in zimbabwe. European Scientific Journal, 11(32).

83. Marcia, J. E. (1966). Development and validation of ego-identity status. Journal of personality and social psychology, 3(5), 551-558.

84. Marcia, J. E. (1967). Ego identity status: Relationship to change in self-esteem, "general maladjustment," and authoritarianism. Journal of Personality, 35, 119-133.

85. Marcia, J. E. (1980). Identity in adolescence. In J. Adelson, Handbook of adolescent psychology. New York: Wiley.

86. McGarty, C., Haslam, S., Hutchinson, K., \& Turner, J. C. (1994). The Effects of Salient Group Memberships on Persuasion. Small Group Research, 25(2), 267-293.

87. McGarty, C., Turner, J., Oakes, P., \& Haslam, S. (1993). The Creation of Uncertainty in the Influence Process: The Roles of Stimulus Information and Disagreement with Similar Others. European Journal of Social Psychology, 23(1), 17-38.

88. Mitchell, T. R., Holtom, B. C., Lee, T. W., \& Sablynski, C. J. (2001). Why people stay: Using organizational embeddedness to predict voluntary turnover. Academy of Management Journal, 44(6), 11021121.

89. Muzio, D., Hodgson, D., Faulconbridge, J., Beaverstock, J., \& Hall, S. (2011). Towards corporate professionalization: the case of project management, management consultancy and executive search. Current Sociology, 59(4), 443-464.

90. Oakes, P. J., Turner, J. C., \& Haslam, S. A. (1991). Perceiving People as Group Members: The Role of Fit in the Salience of Social Categorizations. British Journal of Social Psychology, 30(2), 125-44.

91. Petticrew, M., \& Roberts, H. (2006). How to appraise the studies: an introduction to assessing study quality. In M. Petticrew, \& H. Roberts, Systematic Reviews in the Social Sciences. Hoboken, New Jersey, USA: Wiley-Blackwell.

92. Pierotti, M., Risaliti, G., \& Cestari, G. (2014). Managing IC in an opera house: the case of La Scala. European Scientific Journal, ESJ, 10(34).

93. Porfeli, E. J., Lee, B., Vondracek, F. W., \& Weigold, I. K. (2011). A multi-dimensional measure of vocational identity status. Journal of adolescence, 34(5), 853-871.

94. Preece, D. A., \& Nicol, B. N. (1980). Personnel management, power and the certification process. Personnel Review, 9, 27-32. 
95. Ramarajan, L. (2014). Past, present and future research on multiple identities: Toward an intrapersonal network approach. Academy of Management Annals, 8(1), 589-659.

96. Reicher, S. D., \& Hopkins, N. (2003). On the science of the art of leadership. In D. van Knippenberg, \& M. A. Hogg, Leadership and power: Identity processes in groups and organizations (p. 197-209). London, England: Sage Publications.

97. Reicher, S. D., Haslam, S. A., \& Hopkins, N. (2005). Social identity and the dynamics of leadership: Leaders and followers as collaborative agents in the transformation of social reality. The Leadership Quarterly, 16(4), 547-568.

98. Savickas, M. L. (2005). The theory and practice of career construction. In R. Lent, \& S. D. Brown, Career development and counseling: Putting theory and research to work (p. 42-70). Hoboken, New Jersey: John Wiley \& Sons.

99. Savickas, M. L. (2013). Career construction theory and practice. In R. W. Lent, \& S. D. Brown, Career development and counseling: Putting theory and research to work (p. 147-183). Hoboken, New Jersey: John Wiley \& Sons.

100. Smitina, A. (2010). The link between vocational identity, study choice motivation and satisfaction with studies. Procedia Social and Behavioral Sciences, 5, 1140-1145.

101. Steffens, N. K., Haslam, S. A., \& Reicher, S. D. (2014). Up close and personal: Evidence that shared social identity is a basis for the 'special' relationship that binds followers to leaders. The Leadership Quarterly, 25, 296-313.

102. Strauss, A. L., \& Corbin, J. (1990). Basics of Qualitative Research: Grounded Theory Procedures and Techniques. Newbury Park: SAGE.

103. Sullivan, S., \& Baruch, Y. (2009). Advances in career theory and research: a critical review and agenda for future exploration. Journal of Management, 35(6), 1542- 1571.

104. Tajfel, E., \& Turner, J. C. (1979). An integrative theory of intergroup conflict. In W. G. Austin, \& S. Worchel, The social psychology of intergroup relations (p. 33-47). California: BrooksCole.

105. Tajfel, H., \& Turner, J. C. (1985). The social identity theory of intergroup behavior. In S. Worchel, \& W. Austin, Psychology of intergroup relations. Chicago: Nelson - Hall.

106. Tett, R. P., \& Burnett, D. B. (2003). A personality trait-based interactionist model of job performance. Journal of Applied Psychology, 88(3), 500-517. 
107. Thibaut, J., \& Kelley, H. H. (1959). The social psychology of groups. New York: Wiley.

108. Trope, Y., \& Liberman, N. (2003). Temporal Construal. Psychological Review, 110(3), 403-421.

109. Turban, D. (2001). Organizational attractiveness as an employer on college campuses: an examination of the applicant population. Journal of Vocational Behavior, 58(2), 293-312.

110. Turner, J. (1985). Social categorization and the self concept: a social cognitive theory of group behaviour. In E. Lawler, Advances in Group Processes. Greenwich, CT: JAI Press.

111. Turner, J. C. (1982). Towards a cognitive redefinition of the social group. In H. Tajfel, Social identity and intergroup relations. Cambridge, UK: Cambridge University Press.

112. Turner, J. C., Hogg, M. A., Oakes, P. J., Reicher, S. D., \& Wetherell, M. S. (1987). Rediscovering the social group: A selfcategorization theory. Cambridge, MA: Basil Blackwell.

113. Turner, J., \& Oakes, P. (1997). The Socially Structured Mind. In C. McGarty, \& S. Haslam, The Message of Social Psychology: Perspectives on Mind in Society (p. 355-373). Oxford: Blackwell.

114. Tyler, T. R., \& Blader, S. L. (2002). Autonomous vs. comparative status: Must we be better than others to feel good about ourselves? Organizational Behavior \& Human Decision Processes, 89(1), 813-838.

115. Tyler, T. R., \& Blader, S. L. (2003). The group engagement model: Procedural justice, social identity, and cooperative behavior. Personality and Social Psychology Review, 7(4), 349-361.

116. van Dick, R. (2001). Identification in organizational contexts: Linking theory and research from social and organizational psychology. International Journal of Management Reviews, 3(4), 265-283.

117. Vrontis, D., \& Christofi, M. (2019). R\&D internationalization and innovation: A systematic review, integrative framework and future research directions. Journal of Business Research.

118. Weaver, G. R. (2006). Virtue in organizations: Moral identity as a foundation for moral agency. Organization Studies, 27(3), 341368 . 